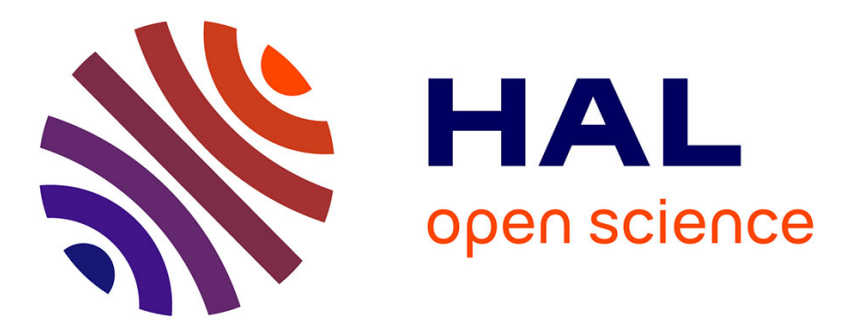

\title{
Atlasing the frontal lobe connections and their variability due to age and education: a spherical deconvolution tractography study
}

K. Rojkova, E. Volle, M. Urbanski, F. Humbert, F. Dell'acqua, Michel Thiebaut de Schotten

\section{To cite this version:}

K. Rojkova, E. Volle, M. Urbanski, F. Humbert, F. Dell'acqua, et al.. Atlasing the frontal lobe connections and their variability due to age and education: a spherical deconvolution tractography study. Brain Structure and Function, 2016, 221 (3), pp.1751-1766. 10.1007/s00429-015-1001-3 . hal01297378

\section{HAL Id: hal-01297378 https://hal.sorbonne-universite.fr/hal-01297378}

Submitted on 11 May 2016

HAL is a multi-disciplinary open access archive for the deposit and dissemination of scientific research documents, whether they are published or not. The documents may come from teaching and research institutions in France or abroad, or from public or private research centers.
L'archive ouverte pluridisciplinaire HAL, est destinée au dépôt et à la diffusion de documents scientifiques de niveau recherche, publiés ou non, émanant des établissements d'enseignement et de recherche français ou étrangers, des laboratoires publics ou privés. 


\section{Atlasing the frontal lobe connections and their variability due to age and education: a spherical deconvolution tractography study.}

Rojkova K. ${ }^{1,2}$, Volle E. ${ }^{1}$, Urbanski M. ${ }^{1,3}$, Humbert F. ${ }^{4}$, Dell'Acqua F. ${ }^{6}$, Thiebaut de Schotten M.*1,2,5

${ }^{1}$ CNRS UMR 7225, Inserm, UPMC-Paris6, UMR_S 1127; CRICM, GH Pitié-Salpêtrière, 75013

Paris, France.

${ }^{2}$ Natbrainlab, Brain and Spine Institute, Paris, France

${ }^{3}$ Service de Médecine et de Réadaptation Gériatrique et Neurologique, Hôpitaux de SaintMaurice, Saint-Maurice, France

${ }^{4}$ Centre de Neuroimagerie de Recherche CENIR, Groupe Hospitalier Pitié-Salpêtrière, Paris, France

${ }^{5}$ Natbrainlab, Sackler Institute of Translational Neurodevelopment, Institute of Psychiatry, King's College London, London, UK

${ }^{6}$ Natbrainlab, Department of Neuroimaging, Institute of Psychiatry, King's College London, London, UK

* Corresponding author: michel.thiebaut@gmail.com 


\section{ABSTRACT:}

In neuroscience, there is a growing consensus that higher cognitive functions may be supported by distributed networks involving different cerebral regions, rather than by single brain areas. Communication within these networks is mediated by white matter tracts and is particularly prominent in the frontal lobes for the control and integration of information. However, the detailed mapping of frontal connections remains incomplete, albeit crucial to an increased understanding of these cognitive functions. Based on forty-seven high-resolution diffusionweighted imaging datasets (age range 22-71), we built a statistical normative atlas of the frontal lobe connections in stereotaxic space, using state of the art spherical deconvolution tractography. We dissected fifty-five tracts including U-shaped fibers. We further characterized these tracts by measuring their correlation with age and education level. We reported age-related differences in the microstructural organization of several, specific frontal fiber tracts, but found no correlation with education level. Future voxel-based analyses, such as voxel-based morphometry or tractbased spatial statistics studies may benefit from our atlas by identifying the tracts and networks involved in frontal functions. Our atlas will also build the capacity of clinicians to further understand the mechanisms involved in brain recovery and plasticity, as well as assist clinicians in the diagnosis of disconnection or abnormality within specific tracts of individual patients with various brain diseases. 
The study of frontal lobe connections is essential to understanding of the role and the organization of the frontal lobes' distinct subregions. The functions of the frontal subregions emerge from an interaction with other cortical areas, on which it exerts its control, and from which it receives information to integrate. These interactions are supported by short and longrange white matter connections. While most of the recent data and theories regarding the functional organization of the frontal lobes have been supported by functional imaging, its anatomical connectivity has yet only been scarcely investigated.

In 1985, a breakthrough came with the emergence of a new magnetic resonance imaging (MRI) modality: the diffusion-weighted MRI (Le Bihan and Breton 1985). This non-invasive technique offers a unique approach to investigate in-vivo the structure of brain tissues by measuring the diffusion of water molecules along different directions. This exciting technical advance not only reproduced reliably known anatomy in the living human brain (Catani et al. 2002; Catani and Thiebaut de Schotten 2008), but also refined the description of large fiber bundles (Catani et al. 2005). Moreover, it also gave raise to studies of intersubject variability (Ciccarelli et al. 2003; Thiebaut de Schotten et al. 2011; Verhoeven et al. 2010; Wakana et al. 2007), asymmetries (Barrick et al. 2007; Catani et al. 2007), behavioral correlates (Barrick et al. 2007; Catani et al. 2007; Johansen-Berg et al. 2007; Thiebaut de Schotten et al. 2014a) and pathological differences (Craig et al. 2009; Thiebaut de Schotten et al. 2014a). While several fiber populations cross almost everywhere in the brain (Dell'Acqua et al. 2013; Jeurissen et al. 2013) standard diffusion tensor tractography models follow the principal direction of fibers, leading to incomplete or erroneous reconstructions of white matter features (Thiebaut de Schotten et al. 2011) that may bias tract-specific measurements (Dell'Acqua and Catani 2012; Vos et al. 
2012). New developments in tractography and diffusion modeling can circumvent this issue by extracting the orientation distribution of different populations of fibers within the same voxel (Dell'acqua et al. 2010; Descoteaux et al. 2007; Tournier et al. 2004; Tuch et al. 2003; Wedeen et al. 2005). These new methods allow for the depiction of tracts that better correspond to postmortem dissections (Dauguet et al. 2007; Dauguet et al. 2006; Lawes et al. 2008; Thiebaut de Schotten et al. 2011) and simian axonal tracing (Thiebaut de Schotten et al. 2012) with good reliability (Kristo et al. 2013b). More recently, a novel nomenclature of the frontal lobe U-shaped connection was made possible through preliminary results that combined post-mortem Klingler dissection (Klingler 1935) with high resolution spherical deconvolution tractography (Catani et al. 2012). However, intersubject variability and aging effects could not be assessed due to the small sample size of participants studied. New tract-specific measurements taking into account the crossing of fibers also followed these advances in diffusion imaging tractography (e.g. Apparent Fiber Density or Hindrance Modulated Orientational Anisotropy (HMOA) Raffelt et al. 2012; Dell'Acqua et al. 2013). These new measurements are specific to the direction followed by the tractography; therefore providing a true-tract specific index to characterize white matter diffusion when crossing. These indices could offer a more precise characterization of the underlying microstructural organization, and consequently, be more sensitive to age-related differences of individual tracts when compared to traditional diffusion tensor imaging indices, such as fractional anisotropy (FA).

The study of the frontal connections is also of particular interest for the neurosciences of aging. With aging, neuronal loss and small vessel alteration lead to progressive and subtle white matter changes associated with cognitive decline in the elderly (Pantoni 2010; Xiong and Mok 2011). Cognitive decline affects predominantly many executive functions but not all (Geerligs et 
al. 2014) and brain changes seem to distribute unevenly, affecting predominantly the frontal region (Bishop et al. 2010; Curiati et al. 2009; Draganski et al. 2011; Giorgio et al. 2010; Good et al. 2001; Moscovitch 1992; Raz et al. 2004; Raz et al. 2000; West 1996) suggesting that latest developed brain areas are especially susceptible to show an accelerated aging (Tamnes et al. 2009). Previous cross sectional studies using tract-specific measurements confirmed this "frontal lobe hypothesis" for the white matter by revealing a slow decrease with aging in the FA for frontal callosal tracts (Hsu et al. 2008; Hasan et al. 2009b; Lebel et al. 2010; Bastin et al. 2008; Michielse et al. 2010) and for long tracts connecting the frontal lobe (Jones et al. 2006; Hasan et al. 2009a; Hasan et al. 2010), affecting more prominently the frontal portion of these tracts (Davis et al. 2009). The same result was corroborated with voxel-based statistics showing a negative correlation between aging and FA in specific but not all areas of the frontal lobes (Giorgio et al. 2010; Madden et al. 2007; Madden et al. 2004; O'Sullivan et al. 2001; Phillips et al. 2013; Raz 2005; Salat et al. 1999). Nevertheless, it is important to note that the "frontal lobe hypothesis" has also been contested by a two years longitudinal study showing no evidence of an accelerated decline in the frontal lobe region in the aging population (Barrick et al. 2010). Aging may affects some, but not all, tracts of frontal white matter. This explains the contrasted results reported in the literature. Age-related differences are not taken into account in atlases built from populations with a restricted age-range; this bias may hamper the use of these atlases in various clinical populations. One way to circumvent this issue is to build a normative global atlas based on a healthy population with a large age range. The effects of age may also differ according to some factors, such as compensatory networks (Stern et al. 2005; Stern et al. 2008) or increased functional connectivity in existing networks (Bastin et al. 2012), providing a partial protection called "cognitive reserve" (Stern 2002, 2009). Higher education seems to also be an important factor delaying aging affects on the brain and cognitive decline (Brayne et al. 2010; Coffey et al. 
1999) and preliminary evidences suggest structural connectivity changes associated with cognitive reserve in elderly (Fischer et al. 2014). However it is unknown whether specific tracts are supporting these changes.

Therefore, the aim of our study was to map, in a sample of healthy participants, all the association, projection and short U-shaped tracts previously described in the frontal lobe. We also estimated the effect of age and level of education on these tracts using measures of the volume, FA and HMOA. The final atlas of the human frontal lobe can be used for future clinicalneuroanatomical correlation in patients of various age ranges and levels of education. 


\section{MATERIAL AND METHODS}

\section{Participants}

This study was approved by the local Ethics Committee. Participants were recruited via advertising in the Salpêtriere hospital and on the web site www.risc.cnrs.fr. All participants were right-handed healthy adults with no history of neurological or psychiatric disorder, no cognitive complaints, and no cognitive impairments or depression, as assessed using translated versions of the Mini Mental state (Folstein et al. 1975) and the MADRS (Montgomery and Asberg 1979). Participants were not included if their MMSE score was lower than 27 and if they have history of neurological or psychiatric symptoms.

All subjects had an MRI scan including 3D T1-weighted images in addition to diffusion images (acquisition, preprocessing, rules for dissections as well as the approach employed to map white matter tracts are reported in supplementary material). All brain images were examined by a neuroradiologist. Millimetric T1-weighted and diffusion weighted images did not show any significant signal abnormalities evocative of a small vessel disease or of an evolving neurological disease. Subjects with abnormalities on MRI were excluded.

Informed and written consent to participate in this study was provided by 57 right-handed volunteers. Participants were excluded from the analysis for medical reasons (anomalies on neuropsychological testing or on the brain MRI) or for head movements during the MRI acquisition. The average age of the 47 remaining participants ( 24 males and 23 females) was 45.45 years ( \pm 14.79 years; aged between 22 and 71 years) and their education level was 15.36 years of education ( \pm 3.00 years; range between 10 and 26 years), noting that a French bachelor's 
degree corresponds to 12 years of education. Subjects from our sample had relatively high education levels, independently of their gender $\left(\mathrm{t}_{(45)}=1.203 ; \mathrm{p}=0.235\right)$ but with a trend of correlation with age, younger participants having a slightly but not significantly higher education level than older participants $(\mathrm{r}=-0.259 ; \mathrm{p}=0.079)$. Therefore partial correlation statistics were carried on controlling for age or education when appropriate.

\section{Statistical analysis}

In order to test whether white matter differences distribute unevenly, affecting predominantly the frontal region, we used SPSS software (SPSS, Inc., Chicago, IL, United States of America) to carry on a repeated ANOVA between tracts emerging from the frontal lobe and tracts not involving the frontal lobe using age and education as covariates.

For each of the following statistics, we employed a False Discovery Rate (FDR) correction (Benjamini and Hochberg 1995) for multiple comparisons; this is available as a tool on the Signed Differential Mapping website (http://www.sdmproject.com/).

Gaussian distribution of the data using the Shapiro-Wilk test (Shapiro and Wilk 1965) was not confirmed for all variables in our group of participants. Therefore, two-tailed partial Spearman rank correlation coefficients (Spearman 1904) were performed between the measurements of each dissected single tract and age, controlling for the education level of the participants. Similarly, partial Spearman ranking correlation coefficients were performed between the measurements of each dissected single tract and the education level controlling for age (FDR corrected for 55 tracts). 


\section{RESULTS}

We successfully identified and mapped, in the MNI reference space, 55 frontal tracts: 16 interlobar association tracts, one commissural tract, eight frontal projection tracts and 30 frontal short U-shaped tracts for both hemispheres (Fig. 1). Maps are provided as an online digital supplementary material (https://www.dropbox.com/s/g0vlklnk60up73y/Atlasmaps.zip) and projections of these tracts are provided in Fig. 2, 3 and 4. Percentage of success for the reconstruction of each tract and tract-specific measurements are tabulated in supplementary table 1. This percentage was calculated using as follows:

Percentage of success $=$ Number of successful reconstructions of the tract/number of participants X 100.

\section{Frontal interlobar association tracts}

\section{Superior longitudinal fasciculus (SLF)}

We reconstructed a system, composing of three parallel longitudinal branches passing through the dorsolateral portion of the white matter, situated above the ventricles and the lateral sulcus, and connecting the parietal and the frontal lobes. The superior branch (SLF I; $\mathrm{n}^{\circ} 1$ in Fig. 1 and Fig. 2) runs from the superior parietal lobule and the precuneus (Brodman Area, BA7 and BA5) to the superior frontal gyrus (BA6, BA8, BA9 up to BA10). More ventrally, the middle branch (SLF II; n² in Fig. 1 and Fig. 2) connects the angular gyrus (BA39) to the posterior regions of the middle frontal gyrus (BA6). Few projections continue further into the middle frontal gyrus up to BA46. The inferior branch (SLF III; n ${ }^{\circ}$ in Fig. 1 and Fig. 2) originates in the 
supramarginal gyrus (BA40) and terminates in the pars opercularis (BA44), triangularis (BA45) and the inferior frontal gyrus (BA47).

Cingulum (n ${ }^{\circ} 4$ in Fig. 1 and Fig. 2)

We identified a long medial fiber bundle running within the cingulate gyrus, all around the corpus callosum, that contains fibers of different lengths. The longest fibers run from the parahippocampal gyrus and the uncus (BA34) to the medial portion of the orbitofrontal (BA11) cortex. The cingulum also contains short U-shaped fibers that connect the medial frontal (BA32, BA8, BA9, BA10) and parietal (BA31, BA5, BA7) to different portions of the cingulate gyrus.

\section{Uncinate ( $n^{\circ} 5$ in Fig. 1 and Fig. 2)}

The fibers of the uncinate originate from the temporal pole (BA38), parahippocampal gyrus, uncus (BA34) and amygdala. After a U-turn, they enter the external capsule then reach the lateral and ventral orbital cortex (BA11, BA12) and frontal pole (BA10).

\section{Arcuate fasciculus}

The fronto-temporal portion of the arcuate fasciculus connects the pars opercularis (i.e. Broca area, BA44) to the auditory cortex (BA22) and the posterior portion of the middle and inferior temporal gyri (BA21, BA37). It forms the long segment (LS; ${ }^{\circ} 6$ in Fig. 1 and Fig. 2) of the arcuate fasciculus, arching around the sylvian fissure. Shorter fronto-parietal fibers link the ventral portion of the precentral gyrus (BA6) to the postcentral gyrus (BA1) and the supramarginal gyrus (BA40). These fibers form the anterior segment (AS; n $^{\circ} 7$ in Fig. 1 and Fig. 2) of the arcuate fasciculus. 
Inferior fronto-occipital fasciculus (IFOF, $n^{\circ} 8$ in Fig. 1 and Fig. 2)

The inferior fronto-occipital fasciculus is a long-ranged bow-tie-shaped tract connecting the occipital lobe to the frontal lobe. It originates from the occipital pole (BA18), the lingual gyrus and the inferior and middle occipital gyri (BA19). As it leaves the occipital lobe, IFOF narrows and its fibers gather at the level of the extreme capsule. As it enters the frontal lobe, its fibers spread to the inferior frontal gyrus. Its ventral fibers reach the orbital gyrus (BA11) and the frontal pole (BA10), whereas its dorsal fibers terminate in the anterior superior frontal gyrus (most anterior part of BA9 and dorsal BA10).

\section{Frontal commissural and projection tracts}

Commissural and projection tracts are difficult to follow with tractography as they intertwine with one another and cross association fibers. Despite spherical deconvolution being designed to circumvent this fiber-crossing issue, their complex configuration may occasionally lead to an underestimation of their true extent. Note that the validity and limitations of these results will be highlighted in the discussion.

Frontal Corpus callosum ( $n^{\circ} 9$ in Fig. 1 and Fig. 3)

The frontal portion of the corpus callosum occupies the rostrum, the genu and most of the body of the corpus callosum and projects on to the whole surface of the frontal lobe except for the pars orbitalis (BA 47). These projections also show a reduced probability for the pars opercularis (BA 44) and the frontal eye field (BA 6).

Corticospinal tract (CST, $n^{\circ} 10$ in Fig. 1 and Fig. 3) 
The corticospinal tract consists of a fan-shaped set of fibers, passing through the posterior limb of the internal capsule and projecting from the precentral gyrus (BA4) to the spinal cord. We note that in a third of participants we obtained its projection onto the whole homunculus in the precentral gyrus; for the remainder of the participants tractography confined its projections to the hand and the lower limb regions.

Fronto-thalamic (anterior thalamic radiations) and fronto-striatal projections ( $n^{\circ} 11$ and 12 in Fig. 1 and Fig. 3)

Fronto-thalamic and fronto-striatal projections pass through the anterior limb of the internal capsule and projects on the whole surface of the frontal lobe. These projections also show a reduced probability for the ventral portion of the precentral gyrus and the middle frontal gyrus (BA 6 and 46).

Fronto-pontine projections ( $n^{\circ} 13$ in Fig. 1 and Fig. 3)

Fronto-pontine fibers pass through the genu and the anterior limb of the internal capsule and projects on to the whole surface of the frontal lobe. As for the fronto-thalamic and frontostriatal projections, we note a reduced probability of the fronto-pontine projection in the precentral gyrus and the middle frontal gyrus (BA 6 and 46).

\section{Frontal U-shaped tracts}

Fronto-parietal U-tracts

We mapped a set of five tracts running beneath the central sulcus from the parietal postcentral gyrus (BA1) to frontal precentral gyrus (BA4), which are organized from dorsal to ventral as follows: paracentral U-shaped tract (n ${ }^{\circ} 14$ in Fig. 1 and Fig. 4), ventral face U-shaped 
tract ( $\mathrm{n}^{\circ} 18$ in Fig. 1 and Fig. 4), and hand superior, middle and inferior U-shaped tracts ( ${ }^{\circ} 15$ to 17 in Fig. 1 and Fig. 4).

Frontal aslant tract (FAT) (n¹9 in Fig. 1 and Fig. 3)

The frontal aslant tract is a large tilted tract linking the supplementary and presupplementary motor area (BA6) with the frontal operculum (BA44) and the posterior portion of the pars triangularis (BA45).

Fronto-insular tracts (FITs) (n॰20 to 24 in Fig. 1 and Fig. 3)

A system composing of five U-shaped tracts dives from the frontal lobe into the external capsule to reach the insula. The four most anterior tracts, 1 to 4 , link respectively the pars orbitalis or BA47 (FIT 1; $\mathrm{n}^{\circ} 20$ in Fig. 1 and Fig. 4), the pars triangularis or BA45 (FIT 2; $\mathrm{n}^{\circ} 21$ in Fig. 1 and Fig. 4), the pars opercularis or BA44 (FIT 3; n 22 in Fig. 1 and Fig. 4) and the precentral gyrus or BA6 (FIT 4; n²3 in Fig. 1 and Fig. 4) of the frontal lobe with the anterior insula (i.e. anterior short gyrus and middle short gyrus, see Cerliani et al. 2012 for a detailed description of the anatomy of the insula). The most posterior tract (FIT 5; n²4 in Fig. 1 and Fig. 4) connects subcentral gyrus (BA43) to the posterior insula (i.e. anterior long gyrus).

\section{Frontal longitudinal system}

Two parallel chains of U-shaped tracts run longitudinally in the frontal lobe: the frontal superior longitudinal tract (FSL; n²5 in Fig. 1 and Fig. 4) and frontal inferior longitudinal tract (FIL; $n^{\circ} 26$ in Fig. 1 and Fig. 4). 
The longest fibers of frontal superior longitudinal tract connect the precentral gyrus to the anterior portion of the superior frontal gyrus, while its shortest fibers link the middle frontal gyrus with the superior frontal gyrus through a series of a chain-like U-shaped fibers.

The longest fibers of frontal inferior longitudinal tract connect the precentral gyrus to the anterior portion of the middle frontal gyrus, while its shortest fibers link the dorsal portion of the pars opercularis and triangularis with the middle frontal gyrus through a series of chain-like Ushaped fibers.

Fronto-orbito-polar and fronto-marginal tracts

Two intralobar tracts can be observed within the frontal pole. The fronto-orbito-polar tract (FOP; n ${ }^{\circ} 7$ in Fig. 1 and Fig. 4) connects the posterior orbital gyrus (BA12) to the anterior orbital gyrus (BA11) and the ventromedial region of the frontal pole.

The fronto-marginal tract (FMT; $n^{\circ} 28$ in Fig. 1 and Fig. 4) runs beneath the frontomarginal sulcus from the medial to the lateral region of the frontal pole (BA10).

\section{Age and level of education effect}

Repeated ANOVA between measurement of streamlines emerging from the frontal lobe and streamlines not involving the frontal lobe using age and education as covariates revealed a significant interaction between HMOA measures of frontal and non-frontal tracts and the age of the participants $\left(\mathrm{F}_{(1,44)}=6.968 ; \mathrm{p}<0.011\right)$. This result demonstrates that microstructural differences related to age are stronger for the tracts emerging from the frontal lobe compared to other tracts. This effect was not significant for FA measure or relative volume measures. 
Results presented in the following section are false discovery rate (FDR) corrected for multiple comparisons. Uncorrected $\mathrm{p}$ values are reported in supplementary table 2. Tract specific volume measurements did not correlate significantly with aging.

Partial correlation analyses revealed that aging was significantly associated with a decrease of FA (Spearman rank coefficient partial correlation $\delta=-0.519, \mathrm{p}<0.001$ ) and HMOA $(\delta=-0.553, \mathrm{p}<0.001)$ in the frontal projections of the corpus callosum. HMOA measures also decreased with aging in the left hemisphere for the frontal inferior longitudinal fasciculus $(\delta=-$ 0.563, p $<0.001)$, the frontal orbito-polar tract $(\delta=-0.552, \mathrm{p}<0.001)$, fronto-insular tract $5(\delta=$ $-0.379, \mathrm{p}=0.047)$, the corticospinal tract $(\delta=-0.442, \mathrm{p}<0.014)$ and fronto-thalamic projections $(\delta=-0.499, \mathrm{p}=0.004)$ (Fig. 5 and 6). No other partial correlation with age was reported as significant.

Aging was also associated with a decrease of HMOA in the right frontal lobe including the SLF I $(\delta=-0.452, \mathrm{p}=0.015)$, SLF III $(\delta=-0.610, \mathrm{p}<0.001)$ branches of the superior longitudinal fasciculus, the corticospinal tract $(\delta=-0.380, \mathrm{p}<0.046)$ and fronto-thalamic projections $(\delta=-0.518, \mathrm{p}=0.003)($ Fig. 5 and 6).

Tract specific volume, FA and HMOA correlations with education level did not survive FDR correction for multiple comparisons.

\section{DISCUSSION}

In this study we employed advanced spherical deconvolution tractography to dissect 55 white matter pathways within the frontal lobes. We produced a probabilistic atlas of the interlobar, commissural, projection and short U-shaped tracts in a population with a large age 
range. All tracts showed a high inter-subject spatial reproducibility (downloadable as supplementary material; https://www.dropbox.com/s/g0vlklnk60up73y/Atlasmaps.zip). We also replicated correlations between age and microstructural measures of the corpus callosum, bilateral corticospinal tract and fronto-thalamic projections. For the first time, the use of spherical deconvolution combined with the use of tract specific HMOA allowed us to report correlations between age and the frontal inferior longitudinal fasciculus, the fronto-insular tract 5 and orbitopolar tract in the left hemisphere and the SLF I and III in the right hemisphere. Education did not correlate with any of the tract-specific measurements. While our maps can be of interest for clinical neuroanatomical correlations and functional imaging studies, the correlation with age provide a normative measure for future studies on diseases affecting various age ranges.

\section{Probabilistic atlas of the interlobar, commissural, projection and short U-shaped tracts}

Our frontal lobe atlas is a probabilistic replication in the stereotaxic space (MNI152) of the original anatomical post-mortem and tractography dissection performed on a single brain and reported by Catani et al. (2012). Compared to previous work (Mori et al. 2008; O'Donnell and Westin 2007; Lawes et al. 2008; Thiebaut de Schotten et al. 2011), this atlas provides probabilistic maps of the three branches of the superior longitudinal fasciculus and many intralobar and U-shaped connections, as well as a full reconstruction of most of the crossing fibers. These maps can be of interest to identify tracts connecting functional activations (Thiebaut de Schotten et al. 2014a) or disconnections caused by strokes (Thiebaut de Schotten et al. 2014b). It may also provide bases for future studies aiming to clarify the very debated anatomo-functional organization of the prefrontal cortex for executive functions and cognitive control. 
Classically, patient studies demonstrate that the prefrontal cortex is critical to executive functions, and adaptive goal-directed behaviors (Azuar et al. 2014; Burgess et al. 2009; Stuss and Knight 2013; Volle et al. 2008). Influent cognitive models such as the working memory model postulate a specialization of retro-rolandic and of prefrontal regions along the superior-inferior axis. In the posterior prefrontal regions, dissociation between the processing of spatial (in the superior part) and verbal (in the inferior part) information has been reported in both functional MRI (Curtis and D'Esposito 2003; Sakai and Passingham 2003; Sala et al. 2003; Smith and Jonides 1999), and lesion studies (Volle et al. 2008; Volle et al. 2013). The superior and inferior longitudinal U-shaped tracts evidenced in the current study may support this spatial and verbal specialization respectively within the frontal lobe. Posteriorly, separate parietal and temporal inputs to these systems via the superior longitudinal and the arcuate fasciculi may also support this functional segregation. The examination of direct relationship between anatomical tracts and functional dissociations would be of high interest for future studies in order to disambiguate the organization of prefrontal cortex for executive functions.

The longitudinal U-shaped tracts are also interesting to consider regarding the proposed postero-anterior serial organization of frontal functions. A U-shaped organization of connections is concordant with caudal-rostral models (Badre and D'Esposito 2007; Badre 2008; Christoff et al. 2009) of lateral prefrontal organization, such as the cascade model of cognitive control (Koechlin and Summerfield 2007; Koechlin et al. 2003; Koechlin et al. 1999). In these models, more posterior regions correspond to a lower-level module and receive signals from a higherlevel module supported by more anterior regions. The processing-level of these modules may depend on the level of cognitive control, of relational complexity or of abstraction of representations. The most anterior region, or rostral prefrontal cortex, is thought to support the 
highest level modules, and has indeed been consistently shown involved in the highest level of cognitive abilities, such as relational reasoning (Smith et al. 2007; Vartanian 2012; Volle et al. 2010), combination of remote information (Gonen-Yaacovi et al. 2013), coordinating goals and subgoals (Koechlin et al. 1999; Kroger et al. 2002) and multitasking (Burgess et al. 2009; Volle et al. 2011). The U-shaped tracts composing the frontal longitudinal system may constitute the anatomical link between successive modules. This hypothesis may be tested in future studies.

It is also interesting to note that projection areas of different tracts are not randomly distributed in the frontal lobes, but seem to overlap in preferred locations, such as the inferior frontal junction, the posterior part of the superior frontal sulcus, the inferior frontal gyrus, and the rostral prefrontal region. These cortical regions have been well observed in a myriad of functional imaging studies, using various cognitive tasks. They may well be functional hubs, in addition to anatomical hubs.

\section{White matter correlations with age and education.}

We report that age-related white matter differences distribute unevenly, inducing subtle differences in several tracts of the frontal region but not all. Our analysis confirms previous evidence of reduced microstructural organization in the frontal portion of the corpus callosum associated with aging (Lebel and Beaulieu 2011; Lebel et al. 2008; Lebel et al. 2010). This commissural decline may explain why increased reaction time is associated with aging, as reported during tasks requiring interhemispheric transfer (Gootjes et al. 2004; Reuter-Lorenz and Stanczak 2000) that may sometimes be compensated by alternative use of a different portion of the corpus callosum (Schulte et al. 2013). Similarly our analysis confirmed the decreased age- 
related microstructural measurements of the left and right corticospinal tract recently associated with age-related differences in fine motor control (Holtrop et al. 2014).

Age-related differences reported in the anterior thalamic radiations bilaterally are concordant with the age-related significant decrease of the neuron number in the anterior thalamic nuclei (Panadero and Gonzalo Sanz 1988). The anterior thalamic radiations are also part of network involving the hippocampus and dedicated to episodic memory (Aggleton et al. 2010; Catani et al. 2013). Hence the decreased age-related microstructural integrity of the anterior thalamic radiation might be related to the normal aging of episodic memory function (Brickman and Stern 2009; Charlton et al. 2010).

We also explored age-related correlations for new pathways not defined in other atlases. For the first time to our knowledge, we reported an age-related microstructural decreased integrity of two interlobar tracts in the right hemisphere (SLF I and III) and three intralobar tracts in the left hemisphere (the frontal inferior longitudinal fasciculus, the frontal orbito-polar tract and the fronto-insular tract 5). The contrast between the two hemispheres suggest that white matter differences related to age are not similar for the left and the right hemispheres and may be supported by different mechanisms.

Education level did not correlate significantly with tract-specific measurements, even when controlling for the effect of age. This finding suggests that education does not change or strengthen the white matter microstructure of the frontal lobe. Compensation mechanisms to counteract neural decline may therefore be supported by other mechanisms such as the recruitment of supplementary "compensatory" brain networks (Stern et al. 2005; Stern et al. 2008; Cabeza et al. 2002; Cabeza and Dennis 2012). Comparing the effect of education or 
measures of general intelligence using task-specific functional connectivity in young adults and elderly may provide further support to this hypothesis.

\section{FA and HMOA tract specific measurements.}

Spherical deconvolution tractography produced larger bundles, including voxels where fibers are crossing, which indubitably decreased FA and may have biased FA measures. New tract-specific measurements, such as HMOA, are less susceptible to crossing fiber effects. Correlations between age and tract-specific HMOA, but not with FA, suggest that HMOA produces a better estimate of white matter bundle microstructure, when crossing with other fiber populations (Dell'Acqua et al. 2013). Therefore, when undertaking tract-specific measurements using advanced fiber-crossing algorithms, it is preferable to use real tract-specific measures, such as HMOA, rather than voxel specific measures, such as FA.

\section{Limitations}

We employed a structure-specific approach to quantify age-related changes occurring within frontal tracts instead of a whole brain voxel based approach. This approach may have missed differences that may occur at the voxel level. However structure-specific approach does not suffer normalization approximations and is therefore more sensitive than analysis performed over the whole brain (Catani 2006). New hybrids approaches, such as the one reported in Yushkevich et al. (Yushkevich et al. 2008), using true-tract specific indices to characterize white 
matter diffusion when crossing might bring the best of structure specific and voxel based approaches.

We noted that volume measurements have a larger standard deviation than FA or HMOA measurements. This is important as a larger standard deviation reduces the statistical power and decreases the chances of reporting a significant result. Accuracy of tract volume measurements depends on the quality of the dissection, which relies on the reliability of the operator and the quality of the dataset. We employed a semi-automatic approach for the dissection in order to reduce variability, and subsequently, all tracts were visually inspected to reduce false positives. However false negatives may still have been the source for this increase in volume variability (Jones et al. 2013; Kristo et al. 2013a).

We also report preliminary results on the distribution of the corpus callosum, corticospinal tract, striatum, pons and thalamus connections to the cortical surface. Axonal tracing studies suggest that a comparable distribution of projection for the corpus callosum (Myers 1965), the striatum (Haber et al., 2000), the thalamus (Barbas et al., 1991) and the pons (Schmahmann and Pandya, 1995) also exists in monkeys. Indeed, the frontal corpus callosum decreases its probability of projection for the frontal eye-field, the orbito frontal gyrus and the frontal operculum in monkey axonal tracing (Myers, 1965) as well as in the map we presented. Similarly, the striatal projection seems to be reduced for the Brodmann areas 9 and 46 (Haber et al. 2000) and lighter projections from the pons were received from the medial and ventrolateral cortices (Schmahmann and Pandya 1995). However, quantitative maps of the fiber density arising from or terminating in the simian cortex are missing in literature and prevent us from drawing firm comparative anatomy conclusions. Furthermore, in most of our participants the corticospinal tract did not connect to the whole motor homunculus. Hence, although spherical 
deconvolution may put forward an encouraging improvement in the quality of the dissections as compared to standard tensor tractography, it is not exempt of limitations. It should be used with caution, especially when reconstructing projection pathways or in clinical applications where edema, tumors, lesions and infarcts are present then multiple compartments and partial volume effects will limit and confound spherical deconvolution (Roine et al. 2014).

Finally, our cross sectional report suggests heterogeneous age-related microstructural differences within the frontal lobe, that ideally, will require replication in a longitudinal study.

\section{Conclusions}

This atlas of the frontal lobe connections may help identifying tracts connecting functional activations thus contributing to a better understanding of frontal functions and processes. It may also help clinicians to diagnose the disconnection or abnormality of specific tracts on patients' MRIs following a wide range of pathologies including but not limited to stroke, degenerative diseases, traumatic brain injury, multiple sclerosis, tumors, vascular malformations, leukoencephalopathies, and infectious diseases. In relation to clinical disorders, our maps could increase understanding of cognitive deficits, as well as mechanisms of brain recovery and plasticity. However, special caution should be taken when overlapping the atlas maps with the structural data of patients due, for example, to tissue displacement and an altered anatomy (Brett et al. 2001; Clark et al. 2003). Finally, tract-specific correlations with aging produce interesting hypotheses on possible early cognitive decline dissociations that may be tested in stroke and elderly patients. 


\section{ACKNOWLEDGMENTS}

We thank Dr. Marco Catani for his assistance with the anatomical dissection and insightful discussions. We also thank the French Agence Nationale de la Recherche for their support of this project (project CAFORPFC, no. ANR-09-RPDOC-004-01 and project PHENOTYPES, no. ANR-13-JSV4-0001-01). In addition, we also thank the program "Investissements d'avenir" (ANR-10-IAIHU-06) for their generous support.

\section{REFERENCE}

Aggleton JP, O'Mara SM, Vann SD, Wright NF, Tsanov M, Erichsen JT (2010) Hippocampalanterior thalamic pathways for memory: uncovering a network of direct and indirect actions. Eur J Neurosci 31 (12):2292-2307.

Azuar C, Reyes P, Slachevsky A, Volle E, Kinkingnehun S, Kouneiher F, Bravo E, Dubois B, Koechlin E, Levy R (2014) Testing the model of caudo-rostral organization of cognitive control in the human with frontal lesions. Neuroimage 84:1053-1060.

Badre D (2008) Cognitive control, hierarchy, and the rostro-caudal organization of the frontal lobes. Trends Cogn Sci 12 (5):193-200.

Badre D, D'Esposito M (2007) Functional magnetic resonance imaging evidence for a hierarchical organization of the prefrontal cortex. J Cogn Neurosci 19 (12):2082-2099.

Barrick TR, Charlton RA, Clark CA, Markus HS (2010) White matter structural decline in normal ageing: a prospective longitudinal study using tract-based spatial statistics. Neuroimage 51 (2):565-577.

Barrick TR, Lawes IN, Mackay CE, Clark CA (2007) White matter pathway asymmetry underlies functional lateralization. Cereb Cortex 17 (3):591-598.

Bastin C, Yakushev I, Bahri MA, Fellgiebel A, Eustache F, Landeau B, Scheurich A, Feyers D, Collette F, Chetelat G, Salmon E (2012) Cognitive reserve impacts on inter-individual variability in resting-state cerebral metabolism in normal aging. Neuroimage 63 (2):713722.

Bastin ME, Piatkowski JP, Storkey AJ, Brown LJ, Maclullich AM, Clayden JD (2008) Tract shape modelling provides evidence of topological change in corpus callosum genu during normal ageing. Neuroimage 43 (1):20-28.

Benjamini Y, Hochberg Y (1995) Controlling the false discovery rate: a practical and powerful approach to multiple testing. Journal of the Royal Statistical Society Series B (Methodological):289-300.

Bishop NA, Lu T, Yankner BA (2010) Neural mechanisms of ageing and cognitive decline. Nature 464 (7288):529-535.

Brayne C, Ince PG, Keage HA, McKeith IG, Matthews FE, Polvikoski T, Sulkava R (2010) Education, the brain and dementia: neuroprotection or compensation? Brain $133(\mathrm{Pt}$ 8):2210-2216. 
Brett M, Leff AP, Rorden C, Ashburner J (2001) Spatial normalization of brain images with focal lesions using cost function masking. Neuroimage 14 (2):486-500.

Brickman AM, Stern Y (2009) Aging and memory in humans. In: L.R. S (ed) Encyclopedia of Neuroscience. Academic Press, Oxford, pp 175-180.

Burgess PW, Alderman N, Volle E, Benoit RG, Gilbert SJ (2009) Mesulam's frontal lobe mystery re-examined. Restor Neurol Neurosci 27:493-506.

Cabeza R, Anderson ND, Locantore JK, McIntosh AR (2002) Aging gracefully: compensatory brain activity in high-performing older adults. Neuroimage 17 (3):1394-1402.

Cabeza R, Dennis N (2012) Frontal lobes and aging: Deterioration and compensation. In: Stuss D, Knight R (eds) Principles of Frontal Lobe Function, 2nd Edition. pp 628-652.

Catani M (2006) Diffusion tensor magnetic resonance imaging tractography in cognitive disorders. Curr Opin Neurol 19 (6):599-606.

Catani M, Allin MP, Husain M, Pugliese L, Mesulam MM, Murray RM, Jones DK (2007) Symmetries in human brain language pathways correlate with verbal recall. Proc Natl Acad Sci U S A 104 (43):17163-17168.

Catani M, Dell'acqua F, Thiebaut de Schotten M (2013) A revised limbic system model for memory, emotion and behaviour. Neurosci Biobehav Rev 37 (8):1724-1737.

Catani M, Dell'acqua F, Vergani F, Malik F, Hodge H, Roy P, Valabregue R, Thiebaut de Schotten M (2012) Short frontal lobe connections of the human brain. Cortex 48 (2):273291.

Catani M, Howard RJ, Pajevic S, Jones DK (2002) Virtual in vivo interactive dissection of white matter fasciculi in the human brain. NeuroImage 17 (1):77-94.

Catani M, Jones DK, ffytche DH (2005) Perisylvian language networks of the human brain. Annals of Neurolology 57 (1):8-16.

Catani M, Thiebaut de Schotten M (2008) A diffusion tensor imaging tractography atlas for virtual in vivo dissections. Cortex 44 (8):1105-1132.

Cerliani L, Thomas RM, Jbabdi S, Siero JC, Nanetti L, Crippa A, Gazzola V, D'Arceuil H, Keysers C (2012) Probabilistic tractography recovers a rostrocaudal trajectory of connectivity variability in the human insular cortex. Human Brain Mapping 33 (9):20052034.

Charlton RA, Barrick TR, Markus HS, Morris RG (2010) The relationship between episodic long-term memory and white matter integrity in normal aging. Neuropsychologia 48 (1):114-122.

Christoff K, Keramatian K, Gordon AM, Smith R, Madler B (2009) Prefrontal organization of cognitive control according to levels of abstraction. Brain Res 1286:94-105.

Ciccarelli O, Toosy AT, Parker GJ, Wheeler-Kingshott CA, Barker GJ, Miller DH, Thompson AJ (2003) Diffusion tractography based group mapping of major white-matter pathways in the human brain. NeuroImage 19 (4):1545-1555.

Clark CA, Barrick TR, Murphy MM, Bell BA (2003) White matter fiber tracking in patients with space-occupying lesions of the brain: a new technique for neurosurgical planning? Neuroimage 20 (3):1601-1608.

Coffey CE, Saxton JA, Ratcliff G, Bryan RN, Lucke JF (1999) Relation of education to brain size in normal aging: implications for the reserve hypothesis. Neurology 53 (1):189-196.

Craig MC, Catani M, Deeley Q, Latham R, Daly E, Kanaan R, Picchioni M, McGuire PK, Fahy T, Murphy DG (2009) Altered connections on the road to psychopathy. Molecular Psychiatry 14 (10):946-953, 907. 
Curiati PK, Tamashiro JH, Squarzoni P, Duran FL, Santos LC, Wajngarten M, Leite CC, Vallada H, Menezes PR, Scazufca M, Busatto GF, Alves TC (2009) Brain structural variability due to aging and gender in cognitively healthy Elders: results from the Sao Paulo Ageing and Health study. AJNR Am J Neuroradiol 30 (10):1850-1856.

Curtis CE, D'Esposito M (2003) Success and failure suppressing reflexive behavior. Journal of cognitive neuroscience 15 (3):409-418.

Dauguet J, Peled S, Berezovskii V, Delzescaux T (2007) Comparison of fiber tracts derived from in-vivo DTI tractography with 3D histological neural tract .... NeuroImage.

Dauguet J, Peled S, Berezovskii V, Delzescaux T, Warfield SK, Born R, Westin CF (2006) 3D histological reconstruction of fiber tracts and direct comparison with diffusion tensor MRI tractography. Medical image computing and computer-assisted intervention : MICCAI International Conference on Medical Image Computing and Computer-Assisted Intervention 9 (Pt 1):109-116.

Davis SW, Dennis NA, Buchler NG, White LE, Madden DJ, Cabeza R (2009) Assessing the effects of age on long white matter tracts using diffusion tensor tractography. NeuroImage 46 (2):530-541.

Dell'Acqua F, Catani M (2012) Structural human brain networks: hot topics in diffusion tractography. Curr Opin Neurol 25 (4):375-383.

Dell'acqua F, Scifo P, Rizzo G, Catani M, Simmons A, Scotti G, Fazio F (2010) A modified damped Richardson-Lucy algorithm to reduce isotropic background effects in spherical deconvolution. Neuroimage 49 (2):1446-1458.

Dell'Acqua F, Simmons A, Williams SC, Catani M (2013) Can spherical deconvolution provide more information than fiber orientations? Hindrance modulated orientational anisotropy, a true-tract specific index to characterize white matter diffusion. Hum Brain Mapp 34 (10):2464-2483.

Descoteaux M, Angelino E, Fitzgibbons S, Deriche R (2007) Regularized, fast, and robust analytical Q-ball imaging. Magn Reson Med 58 (3):497-510.

Draganski B, Ashburner J, Hutton C, Kherif F, Frackowiak RS, Helms G, Weiskopf N (2011) Regional specificity of MRI contrast parameter changes in normal ageing revealed by voxel-based quantification (VBQ). Neuroimage 55 (4):1423-1434.

Fischer FU, Wolf D, Scheurich A, Fellgiebel A (2014) Association of structural global brain network properties with intelligence in normal aging. PLoS One 9 (1):e86258.

Folstein MF, Folstein SE, McHugh PR (1975) "Mini-mental state". A practical method for grading the cognitive state of patients for the clinician. J Psychiatr Res 12 (3):189-198.

Geerligs L, Saliasi E, Maurits NM, Renken RJ, Lorist MM (2014) Brain mechanisms underlying the effects of aging on different aspects of selective attention. Neuroimage 91:52-62.

Giorgio A, Santelli L, Tomassini V, Bosnell R, Smith S, De Stefano N, Johansen-Berg H (2010) Age-related changes in grey and white matter structure throughout adulthood. Neuroimage 51 (3):943-951.

Gonen-Yaacovi G, de Souza LC, Levy R, Urbanski M, Josse G, Volle E (2013) Rostral and caudal prefrontal contribution to creativity: a meta-analysis of functional imaging data. Front Hum Neurosci 7:465.

Good CD, Johnsrude IS, Ashburner J, Henson RN, Friston KJ, Frackowiak RS (2001) Cerebral asymmetry and the effects of sex and handedness on brain structure: a voxel-based morphometric analysis of 465 normal adult human brains. NeuroImage 14 (3):685-700.

Gootjes L, Van Strien JW, Bouma A (2004) Age effects in identifying and localising dichotic stimuli: a corpus callosum deficit? J Clin Exp Neuropsychol 26 (6):826-837. 
Haber SN, Fudge JL, McFarland NR (2000) Striatonigrostriatal pathways in primates form an ascending spiral from the shell to the dorsolateral striatum. J Neurosci 20 (6):2369-2382.

Hasan KM, Iftikhar A, Kamali A, Kramer LA, Ashtari M, Cirino PT, Papanicolaou AC, Fletcher JM, Ewing-Cobbs L (2009a) Development and aging of the healthy human brain uncinate fasciculus across the lifespan using diffusion tensor tractography. Brain Res 1276:67-76.

Hasan KM, Kamali A, Abid H, Kramer LA, Fletcher JM, Ewing-Cobbs L (2010) Quantification of the spatiotemporal microstructural organization of the human brain association, projection and commissural pathways across the lifespan using diffusion tensor tractography. Brain Struct Funct 214 (4):361-373.

Hasan KM, Kamali A, Iftikhar A, Kramer LA, Papanicolaou AC, Fletcher JM, Ewing-Cobbs L (2009b) Diffusion tensor tractography quantification of the human corpus callosum fiber pathways across the lifespan. Brain Res 1249:91-100.

Holtrop JL, Loucks TM, Sosnoff JJ, Sutton BP (2014) Investigating Age-related changes in fine motor control across different effectors and the impact of white matter integrity. Neuroimage 96:81-87.

Hsu JL, Leemans A, Bai CH, Lee CH, Tsai YF, Chiu HC, Chen WH (2008) Gender differences and age-related white matter changes of the human brain: a diffusion tensor imaging study. Neuroimage 39 (2):566-577.

Jeurissen B, Leemans A, Tournier JD, Jones DK, Sijbers J (2013) Investigating the prevalence of complex fiber configurations in white matter tissue with diffusion magnetic resonance imaging. Hum Brain Mapp 34 (11):2747-2766.

Johansen-Berg H, Della-Maggiore V, Behrens TEJ, Smith SM, Paus T (2007) Integrity of white matter in the corpus callosum correlates with bimanual co-ordination skills. NeuroImage 36 Suppl 2:T16-21.

Jones DK, Catani M, Pierpaoli C, Reeves SJ, Shergill SS, O'Sullivan M, Golesworthy P, McGuire P, Horsfield MA, Simmons A, Williams SC, Howard RJ (2006) Age effects on diffusion tensor magnetic resonance imaging tractography measures of frontal cortex connections in schizophrenia. Hum Brain Mapp 27 (3):230-238.

Jones DK, Knosche TR, Turner R (2013) White matter integrity, fiber count, and other fallacies: the do's and don'ts of diffusion MRI. Neuroimage 73:239-254.

Klingler J (1935) Erleichterung der makroskopischen Präparation des Gehirn durch den Gefrierprozess. Schweiz Arch Neurol Psychiat 36:247-256.

Koechlin E, Basso G, Pietrini P, Panzer S, Grafman J (1999) The role of the anterior prefrontal cortex in human cognition. Nature 399 (6732):148-151.

Koechlin E, Ody C, Kouneiher F (2003) The architecture of cognitive control in the human prefrontal cortex. Science 302 (5648):1181-1185.

Koechlin E, Summerfield C (2007) An information theoretical approach to prefrontal executive function. Trends Cogn Sci 11 (6):229-235.

Kristo G, Leemans A, de Gelder B, Raemaekers M, Rutten GJ, Ramsey N (2013a) Reliability of the corticospinal tract and arcuate fasciculus reconstructed with DTI-based tractography: implications for clinical practice. Eur Radiol 23 (1):28-36.

Kristo G, Leemans A, Raemaekers M, Rutten GJ, de Gelder B, Ramsey NF (2013b) Reliability of two clinically relevant fiber pathways reconstructed with constrained spherical deconvolution. Magn Reson Med 70 (6):1544-1556.

Kroger JK, Sabb FW, Fales CL, Bookheimer SY, Cohen MS, Holyoak KJ (2002) Recruitment of anterior dorsolateral prefrontal cortex in human reasoning: a parametric study of relational complexity. Cereb Cortex 12 (5):477-485. 
Lawes INC, Barrick TR, Murugam V, Spierings N, Evans DR, Song M, Clark CA (2008) Atlasbased segmentation of white matter tracts of the human brain using diffusion tensor tractography and comparison with classical dissection. NeuroImage 39 (1):62-79.

Le Bihan D, Breton E (1985) Imagerie de diffusion in-vivo par résonance magnétique nucléaire. Comptes rendus de l'Académie des sciences 301:1109-1112.

Lebel C, Beaulieu C (2011) Longitudinal development of human brain wiring continues from childhood into adulthood. J Neurosci 31 (30):10937-10947.

Lebel C, Caverhill-Godkewitsch S, Beaulieu C (2010) Age-related regional variations of the corpus callosum identified by diffusion tensor tractography. Neuroimage 52 (1):20-31.

Lebel C, Walker L, Leemans A, Phillips L, Beaulieu C (2008) Microstructural maturation of the human brain from childhood to adulthood. Neuroimage 40 (3):1044-1055.

Madden DJ, Spaniol J, Whiting WL, Bucur B, Provenzale JM, Cabeza R, White LE, Huettel SA (2007) Adult age differences in the functional neuroanatomy of visual attention: a combined fMRI and DTI study. Neurobiol Aging 28 (3):459-476.

Madden DJ, Whiting WL, Huettel SA, White LE, MacFall JR, Provenzale JM (2004) Diffusion tensor imaging of adult age differences in cerebral white matter: relation to response time. Neuroimage 21 (3):1174-1181.

Michielse S, Coupland N, Camicioli R, Carter R, Seres P, Sabino J, Malykhin N (2010) Selective effects of aging on brain white matter microstructure: a diffusion tensor imaging tractography study. Neuroimage 52 (4):1190-1201.

Montgomery SA, Asberg M (1979) A new depression scale designed to be sensitive to change. Br J Psychiatry 134:382-389.

Mori S, Oishi K, Jiang H, Li X, Akhter K, Hua K, Faria AV, Mahmood A, Woods R, Toga AW, Pike GB, Neto PR, Evans A, Zhang J, Huang H, Miller MI, van Zijl PC, Mazziotta J (2008) Stereotaxic white matter atlas based on diffusion tensor imaging in an ICBM template. NeuroImage 40 (2):570-582.

Moscovitch M (1992) Memory and Working-with-Memory: A Component Process Model Based on Modules and Central Systems. J Cogn Neurosci 4 (3):257-267.

Myers RE (1965) Organization of forebrain commissures. In: Ettlinger EG (ed) Functions of the Corpus Callosum. CIBA Foundation Study Group 20, London, pp 133-143.

O'Donnell LJ, Westin CF (2007) Automatic tractography segmentation using a high-dimensional white matter atlas. IEEE Trans Med Imaging 26 (11):1562-1575.

O'Sullivan M, Jones DK, Summers PE, Morris RG, Williams SC, Markus HS (2001) Evidence for cortical "disconnection" as a mechanism of age-related cognitive decline. Neurology 57 (4):632-638.

Panadero A, Gonzalo Sanz LM (1988) [Memory and aging: changes in the mammillary body and anterior thalamic nuclei due to age]. Revista de medicina de la Universidad de Navarra 32 (4):191-200.

Pantoni L (2010) Cerebral small vessel disease: from pathogenesis and clinical characteristics to therapeutic challenges. Lancet Neurol 9 (7):689-701.

Phillips OR, Clark KA, Luders E, Azhir R, Joshi SH, Woods RP, Mazziotta JC, Toga AW, Narr KL (2013) Superficial white matter: effects of age, sex, and hemisphere. Brain connectivity 3 (2): 146-159.

Raffelt D, Tournier JD, Rose S, Ridgway GR, Henderson R, Crozier S, Salvado O, Connelly A (2012) Apparent Fibre Density: a novel measure for the analysis of diffusion-weighted magnetic resonance images. Neuroimage 59 (4):3976-3994. 
Raz N (2005) The aging brain observed in vivo: differential changes and their modifiers, in: Cognitive Neuroscience of Aging: Linking Cognitive and Cerebral Aging. In: Cabeza R, Nyberg L (eds) Cognitive Neuroscience of Aging. Park DC, New-York, pp 19-57.

Raz N, Gunning-Dixon F, Head D, Rodrigue KM, Williamson A, Acker JD (2004) Aging, sexual dimorphism, and hemispheric asymmetry of the cerebral cortex: replicability of regional differences in volume. Neurobiol Aging 25 (3):377-396.

Raz N, Williamson A, Gunning-Dixon F, Head D, Acker JD (2000) Neuroanatomical and cognitive correlates of adult age differences in acquisition of a perceptual-motor skill. Microscopy research and technique 51 (1):85-93.

Reuter-Lorenz PA, Stanczak L (2000) Differential effects of aging on the functions of the corpus callosum. Developmental neuropsychology 18 (1):113-137.

Roine T, Jeurissen B, Perrone D, Aelterman J, Leemans A, Philips W, Sijbers J (2014) Isotropic non-white matter partial volume effects in constrained spherical deconvolution. Frontiers in neuroinformatics 8:28.

Sakai K, Passingham RE (2003) Prefrontal interactions reflect future task operations. Nat Neurosci 6 (1):75-81.

Sala JB, Rämä P, Courtney SM (2003) Functional topography of a distributed neural system for spatial and nonspatial information maintenance in working memory. Neuropsychologia $41(3): 341-356$.

Salat DH, Kaye JA, Janowsky JS (1999) Prefrontal gray and white matter volumes in healthy aging and Alzheimer disease. Arch Neurol 56 (3):338-344.

Schmahmann JD, Pandya DN (1995) Prefrontal cortex projections to the basilar pons in rhesus monkey: implications for the cerebellar contribution to higher function. Neuroscience letters 199 (3):175-178.

Schulte T, Maddah M, Muller-Oehring EM, Rohlfing T, Pfefferbaum A, Sullivan EV (2013) Fiber tract-driven topographical mapping (FTTM) reveals microstructural relevance for interhemispheric visuomotor function in the aging brain. Neuroimage 77:195-206.

Shapiro S, Wilk M (1965) An Analysis of Variance Test for Normality (Complete Samples). Biometrika 52 (3/4):591-611.

Smith EE, Jonides J (1999) Storage and executive processes in the frontal lobes. Science 283 (5408):1657-1661.

Smith R, Keramatian K, Christoff K (2007) Localizing the rostrolateral prefrontal cortex at the individual level. Neuroimage 36 (4):1387-1396.

Spearman C (1904) The proof and measurement of association between two things. Am J Psychol $15(1): 441-471$.

Stern Y (2002) What is cognitive reserve? Theory and research application of the reserve concept. Journal of the International Neuropsychological Society 8:448-460.

Stern Y (2009) Cognitive reserve. Neuropsychologia 47 (10):2015-2028.

Stern Y, Habeck C, Moeller J, Scarmeas N, Anderson KE, Hilton HJ, Flynn J, Sackeim H, van Heertum R (2005) Brain networks associated with cognitive reserve in healthy young and old adults. Cereb Cortex 15 (4):394-402.

Stern Y, Zarahn E, Habeck C, Holtzer R, Rakitin BC, Kumar A, Flynn J, Steffener J, Brown T (2008) A common neural network for cognitive reserve in verbal and object working memory in young but not old. Cereb Cortex 18 (4):959-967.

Stuss DT, Knight RT (2013) Principles of Frontal Lobe Function. Oxford University Press. 
Tamnes CK, Ostby Y, Fjell AM, Westlye LT, Due-Tonnessen P, Walhovd KB (2009) Brain Maturation in Adolescence and Young Adulthood: Regional Age-Related Changes in Cortical Thickness and White Matter Volume and Microstructure. Cereb Cortex.

Thiebaut de Schotten M, Cohen L, Amemiya E, Braga LW, Dehaene S (2014a) Learning to read improves the structure of the arcuate fasciculus. Cereb Cortex 24 (4):989-995.

Thiebaut de Schotten M, Dell'acqua F, Valabregue R, Catani M (2012) Monkey to human comparative anatomy of the frontal lobe association tracts. Cortex 48:82-96.

Thiebaut de Schotten M, Ffytche DH, Bizzi A, Dell'Acqua F, Allin M, Walshe M, Murray R, Williams SC, Murphy DG, Catani M (2011) Atlasing location, asymmetry and intersubject variability of white matter tracts in the human brain with MR diffusion tractography. Neuroimage 54 (1):49-59.

Thiebaut de Schotten M, Tomaiuolo F, Aiello M, Merola S, Silvetti M, Lecce F, Bartolomeo P, Doricchi F (2014b) Damage to white matter pathways in subacute and chronic spatial neglect: a group study and 2 single-case studies with complete virtual "in vivo" tractography dissection. Cereb Cortex 24 (3):691-706.

Tournier JD, Calamante F, Gadian DG, Connelly A (2004) Direct estimation of the fiber orientation density function from diffusion-weighted MRI data using spherical deconvolution. NeuroImage 23 (3):1176-1185.

Tuch DS, Reese TG, Wiegell MR, Wedeen VJ (2003) Diffusion MRI of complex neural architecture. Neuron 40 (5):885-895.

Vartanian O (2012) Dissociable neural systems for analogy and metaphor: implications for the neuroscience of creativity. British journal of psychology 103 (3):302-316.

Verhoeven JS, Sage CA, Leemans A, Van Hecke W, Callaert D, Peeters R, De Cock P, Lagae L, Sunaert S (2010) Construction of a stereotaxic DTI atlas with full diffusion tensor information for studying white matter maturation from childhood to adolescence using tractography-based segmentations. Hum Brain Mapp 31 (3):470-486.

Volle E, Gilbert SJ, Benoit RG, Burgess PW (2010) Specialization of the rostral prefrontal cortex for distinct analogy processes. Cereb Cortex 20 (11):2647-2659.

Volle E, Gonen-Yaacovi G, Costello Ade L, Gilbert SJ, Burgess PW (2011) The role of rostral prefrontal cortex in prospective memory: a voxel-based lesion study. Neuropsychologia 49 (8):2185-2198.

Volle E, Kinkingnehun S, Pochon JB, Mondon K, Thiebaut de Schotten M, Seassau M, Duffau H, Samson Y, Dubois B, Levy R (2008) The functional architecture of the left posterior and lateral prefrontal cortex in humans. Cereb Cortex 18 (10):2460-2469.

Volle E, Levy R, Burgess PW (2013) A new era for lesion-behavior mapping of prefrontal functions. In: D.T. S, Knight RT (eds) Principles of Frontal Lobe Function. pp 500-523.

Vos SB, Jones DK, Jeurissen B, Viergever MA, Leemans A (2012) The influence of complex white matter architecture on the mean diffusivity in diffusion tensor MRI of the human brain. Neuroimage 59 (3):2208-2216.

Wakana S, Caprihan A, Panzenboeck MM, Fallon JH, Perry M, Gollub RL, Hua K, Zhang J, Jiang H, Dubey P, Blitz A, van Zijl PC, Mori S (2007) Reproducibility of quantitative tractography methods applied to cerebral white matter. NeuroImage 36 (3):630-644.

Wedeen VJ, Hagmann P, Tseng W-YI, Reese TG, Weisskoff RM (2005) Mapping complex tissue architecture with diffusion spectrum magnetic resonance imaging. Magnetic resonance in medicine : official journal of the Society of Magnetic Resonance in Medicine / Society of Magnetic Resonance in Medicine 54 (6):1377-1386. 
West RL (1996) An application of prefrontal cortex function theory to cognitive aging. Psychological bulletin 120 (2):272-292.

Xiong YY, Mok V (2011) Age-related white matter changes. J Aging Res 2011:617927.

Yushkevich PA, Zhang H, Simon TJ, Gee JC (2008) Structure-specific statistical mapping of white matter tracts. Neuroimage 41 (2):448-461. 


\section{CAPTIONS}

Fig. 1. Spherical deconvolution tractography of frontal white matter connections. For each tract a single participant map is supplied as a representative example of the individual anatomy. First (SLF I, light blue, $n^{\circ} 1$ ), second (SLF II, dark blue, $n^{\circ} 2$ ) and third (SLF III, pink, ${ }^{\circ} 3$ ) branches of the superior longitudinal fasciculus; cingulum (yellow, $n^{\circ} 4$ ); uncinate (pink, $n^{\circ} 5$ ); long (LS, red, $n^{\circ} 6$ ) and anterior (AS, green, $n^{\circ} 7$ ) segments of the arcuate fasciculus; inferior frontooccipital fasciculus (IFOF, $\mathrm{n}^{\circ} 8$ ); frontal corpus callosum (CC, $\left.{ }^{\circ} 9\right)$; corticospinal tract (CST, $\mathrm{n}^{\circ} 10$ ); fronto-thalamic projections or anterior thalamic radiations (blue, $\mathrm{n}^{\circ} 11$ ); fronto-striatal projections (yellow, $n^{\circ} 12$ ); fronto-pontine projections $\left(n^{\circ} 13\right)$; paracentral $U$ tract (pink, $\left.n^{\circ} 14\right)$; hand superior (green, $\mathrm{n}^{\circ} 15$ ), middle (yellow, $\mathrm{n}^{\circ} 16$ ) and inferior (red, $\mathrm{n}^{\circ} 17$ ) $\mathrm{U}$ tract; face $\mathrm{U}$ tract (blue, $n^{\circ} 18$ ); frontal aslant tract (pink, $n^{\circ} 19$ ); fronto insular tract 1 (FIT 1, light blue, $n^{\circ} 20$ ), 2 (FIT 2, dark blue, $n^{\circ} 21$ ), 3 (FIT 3, red, n²2), 4 (FIT 4, yellow, n²3) and 5 (FIT 5, green, n²4); frontal superior longitudinal (FSL, light blue $\mathrm{n}^{\circ} 25$ ); frontal inferior longitudinal (FIL, pink, $\left.\mathrm{n}^{\circ} 26\right)$, frontal orbito-polar tract (FOP, yellow, $\left.\mathrm{n}^{\circ} 27\right)$, fronto-marginal tract $\left(\mathrm{FMT}\right.$, red, $\left.\mathrm{n}^{\circ} 28\right)$.

Fig. 2. Cortical projection in the MNI stereotaxic space of the frontal interlobar tracts [top to bottom: the three branches of the superior longitudinal fasciculus (SLFI, SLFII and SLFIII), the cingulum, the uncinate, the arcuate long segment (arcuate LS), the arcuate anterior segment (arcuate AS) and the inferior fronto-occipital fasciculus (IFOF)]. Color brightness depends on overlap percentage. A $50 \%$ overlap in a voxel means that tract is present in $50 \%$ of participants for this voxel. The numbers on the tridimensional reconstruction, correspond to Brodmann areas. 
Fig. 3. Cortical projection in the MNI stereotaxic space of the commissural and projection tracts. The numbers located beneath the figure refer to the MNI coordinates of different slices displayed. We used a rainbow color code to indicate the percentage overlap. The numbers displayed on the tridimensional reconstruction, correspond to Brodmann areas.

Fig. 4. Cortical projection in the MNI stereotaxic space of frontal short U-shaped tracts (top to bottom: paracentral, hand superior, middle and inferior, face U-shaped, frontal aslant, fronto insular 1, 2, 3, 4, 5, frontal superior longitudinal, frontal inferior longitudinal, fronto-orbito-polar and fronto-marginal tracts). The numbers located beneath the figure refer to the MNI coordinates of different slices displayed. Color brightness depends on overlap percentage. The numbers displayed on the tridimensional reconstruction, correspond to Brodmann areas.

Fig. 5. Correlation between the participants' age and tract-specific measurements. Participants with higher education are represented as a red dot and participants with a lower education as a blue dot a) frontal corpus callosum b) left and right corticospinal tract; c) left and right anterior thalamic radiations. $* \mathrm{p}<0.05, * * \mathrm{p}<0.01, * * * \mathrm{p}<0.001$ false discovery rate corrected for multiple comparison. For each tract a single participant $3 \mathrm{D}$ reconstruction is supplied as a representative example of the individual anatomy.

Fig. 6. Correlation between the participants' age and tract-specific measurements. Participants with higher education are represented as a red dot and participants with a lower education as a blue dot a) first branch of the right superior longitudinal fasciculus; b) third branch of the right superior longitudinal fasciculus; c) fronto-insular tract 5; d) left frontal orbito-polar tract; e) left frontal inferior longitudinal fasciculus. ${ }^{*} \mathrm{p}<0.05, * * \mathrm{p}<0.01, * * * \mathrm{p}<0.001$ false discovery 
rate corrected for multiple comparison. For each tract a single participant $3 \mathrm{D}$ reconstruction is supplied as a representative example of the individual anatomy. 


\section{FRONTAL ASSOCIATION TRACTS}
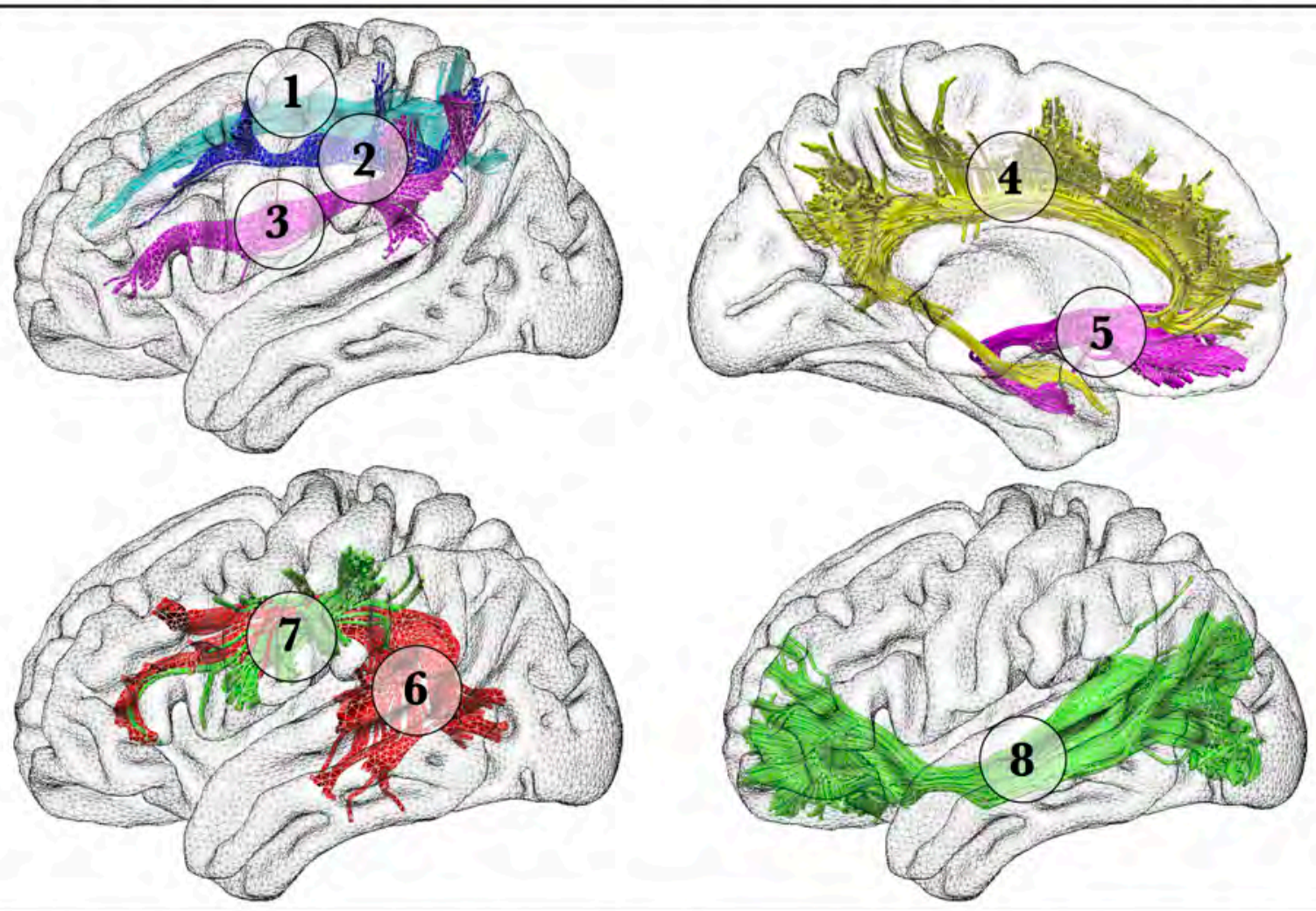

\section{FRONTAL COMMISSURAL TRACT}

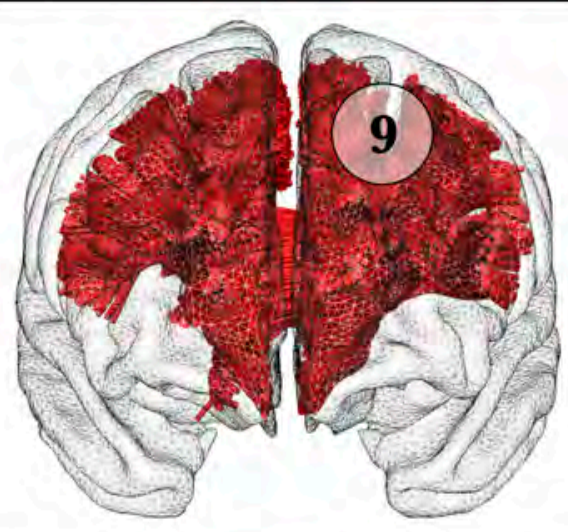

\section{FRONTAL PROJEGTION TRAGTS}
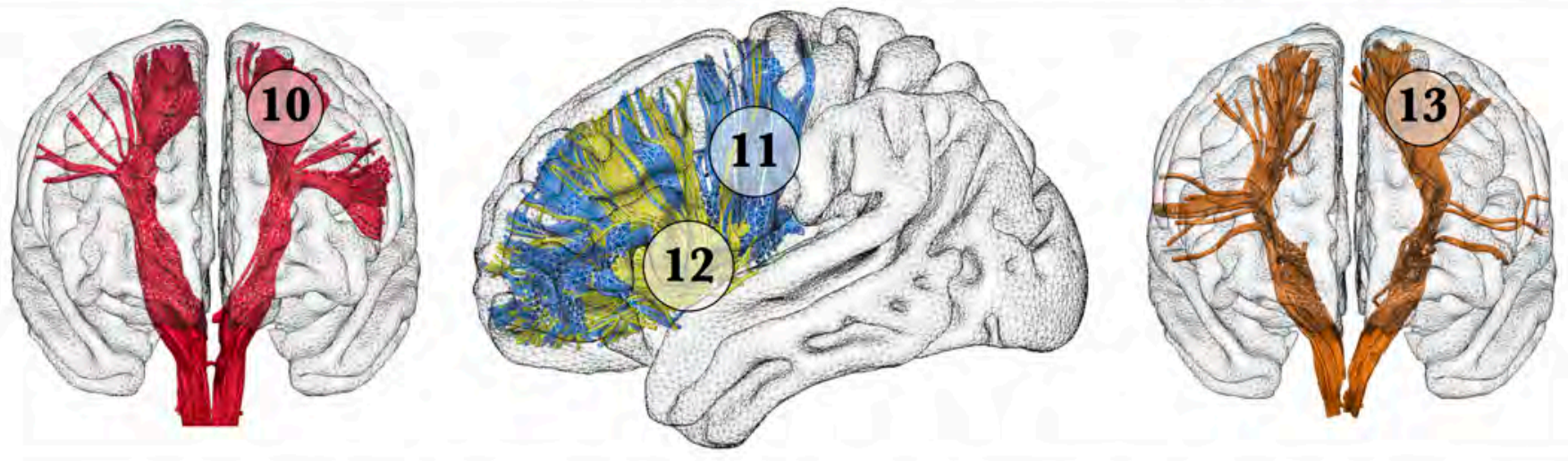

\section{FRONTAL U-SHAPED TRACTS}

1415
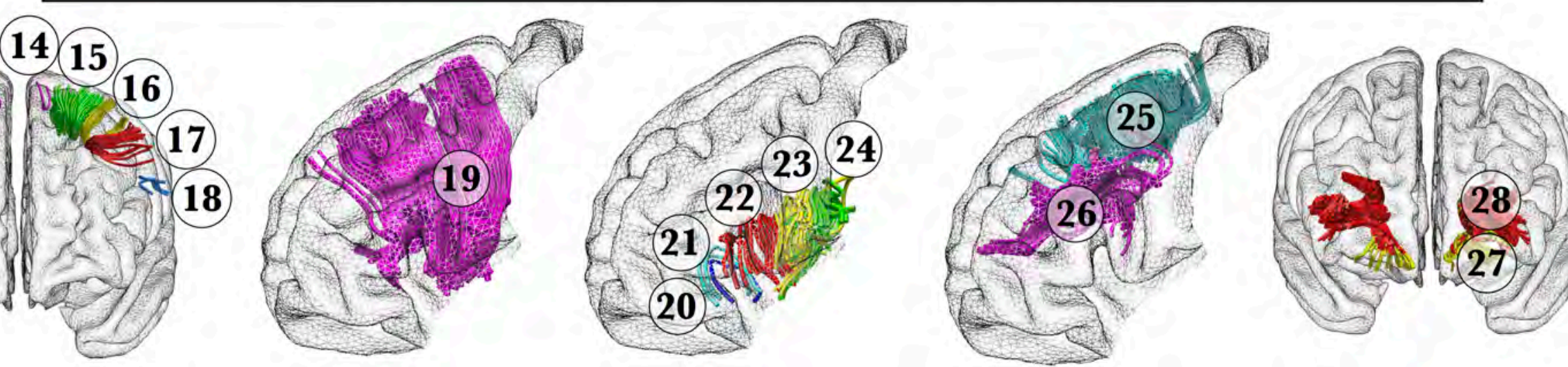


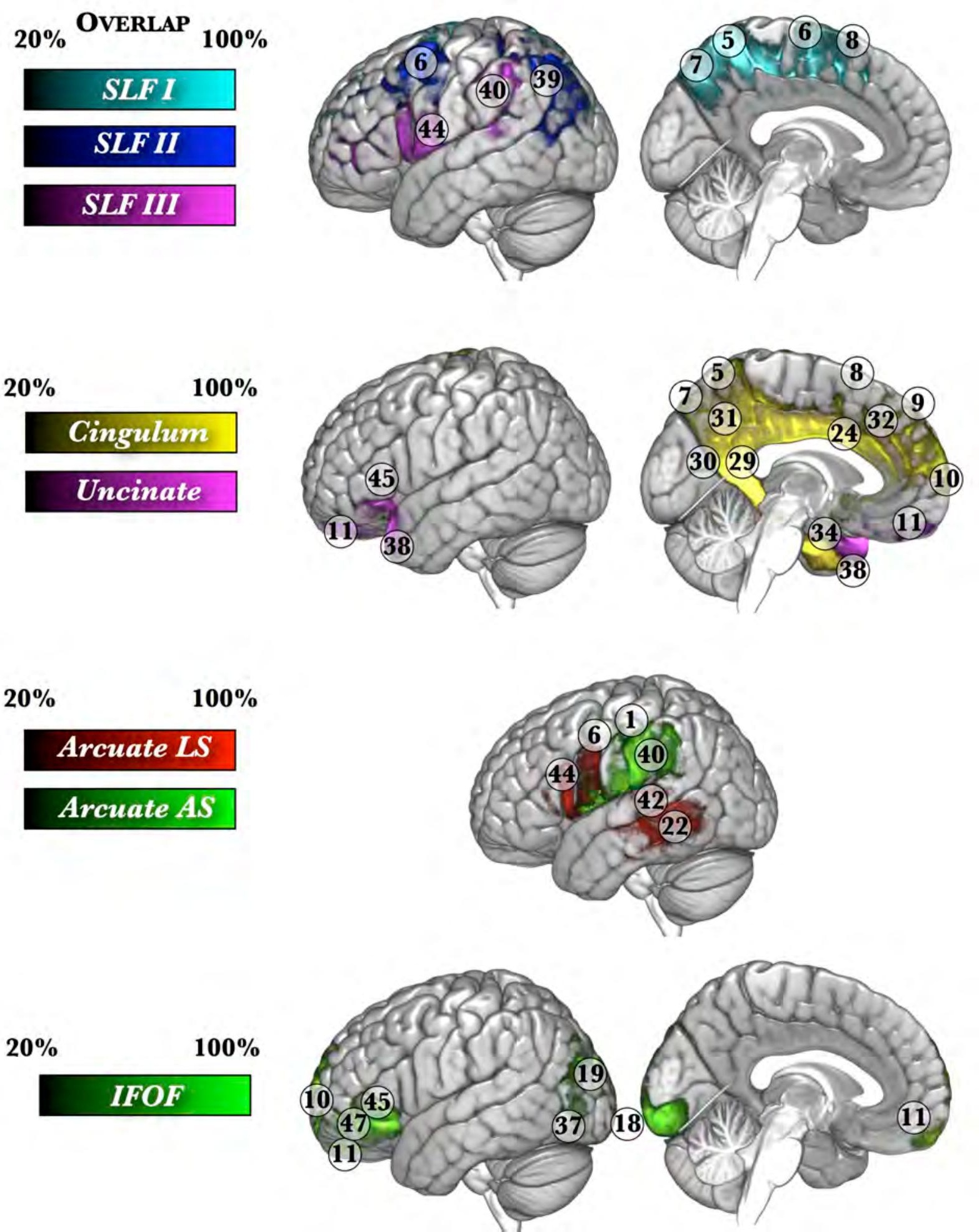




\section{OVERLAP}

$20 \% \quad 100 \%$

Paracentral

\begin{tabular}{l} 
Hand sup. \\
Hand mid. \\
\hline
\end{tabular}

\section{Hand inf.}

Face $U$
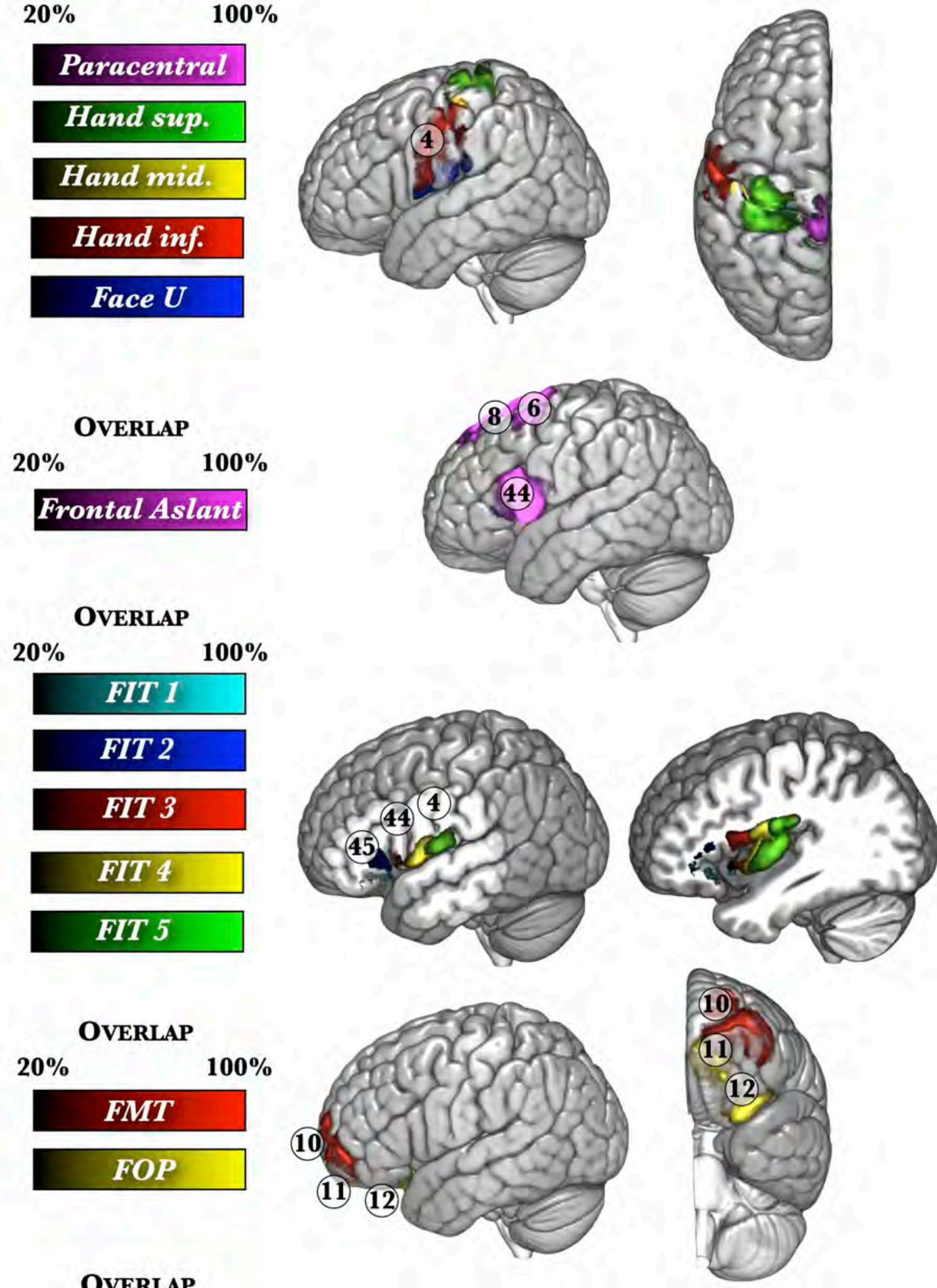

OVERLAP

\begin{tabular}{|c|}
\hline FSL \\
\hline FIL \\
\hline
\end{tabular}

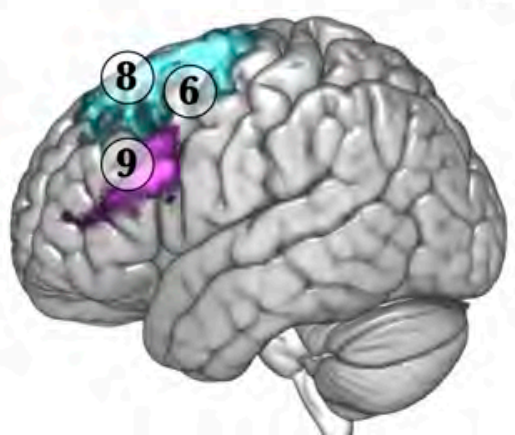


a)
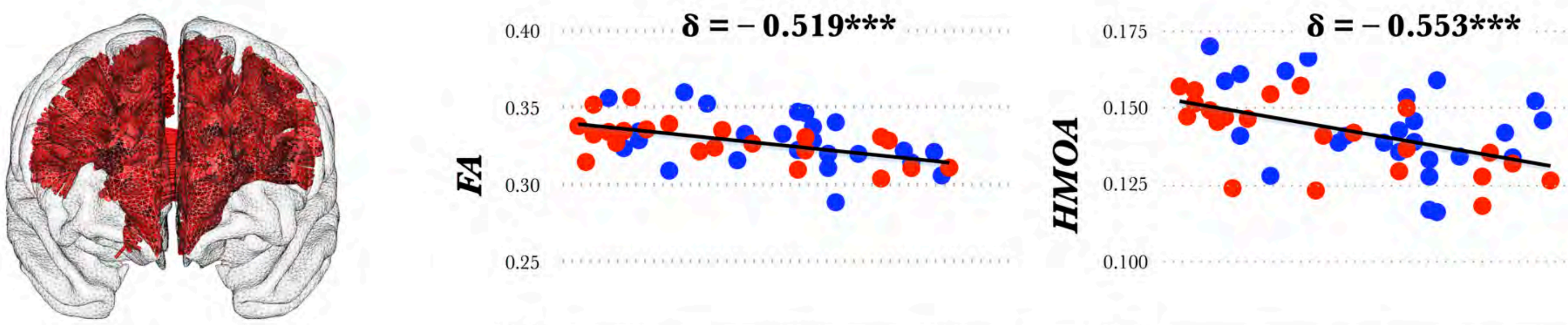

0.25

0.100
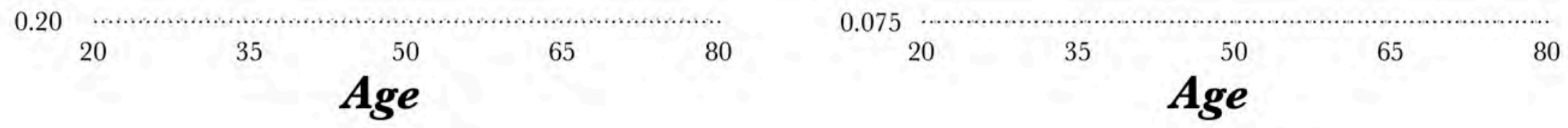

0.175 left hemisphere $\delta=-\mathbf{0 . 4 4 2 *}$

0.175 right hemisphere $\delta=-\mathbf{0 . 3 8 0}$ *

b)
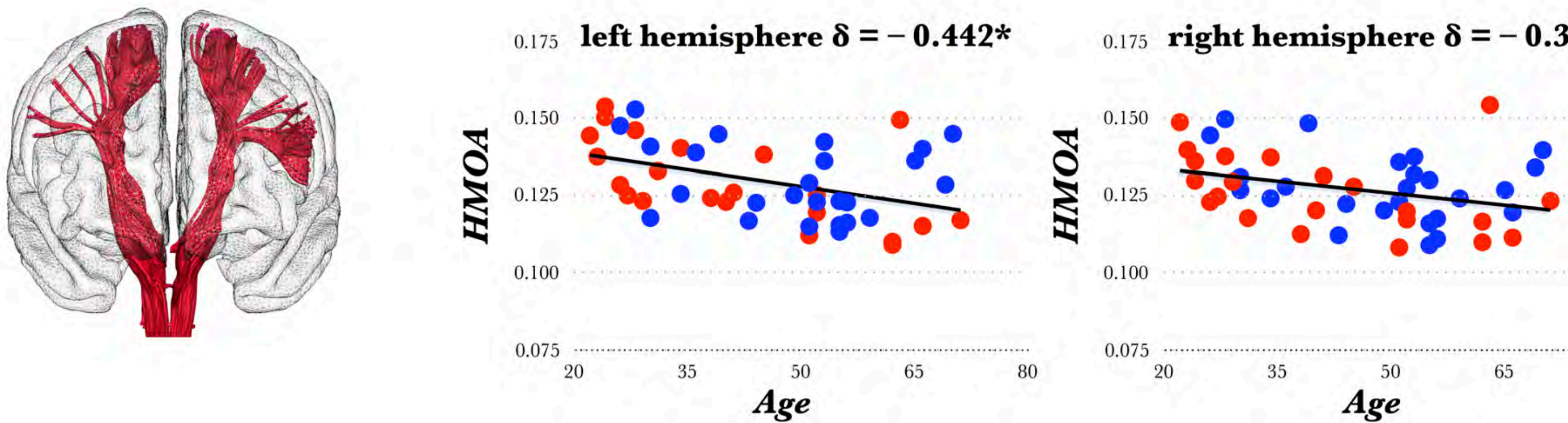

0.100

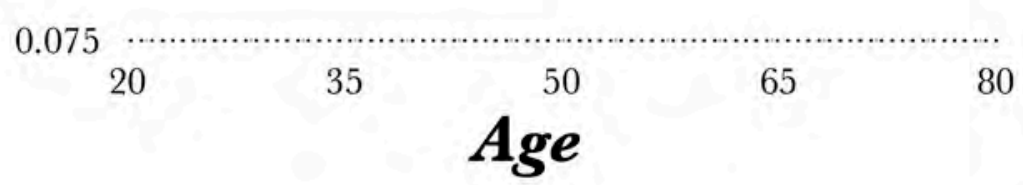

c)

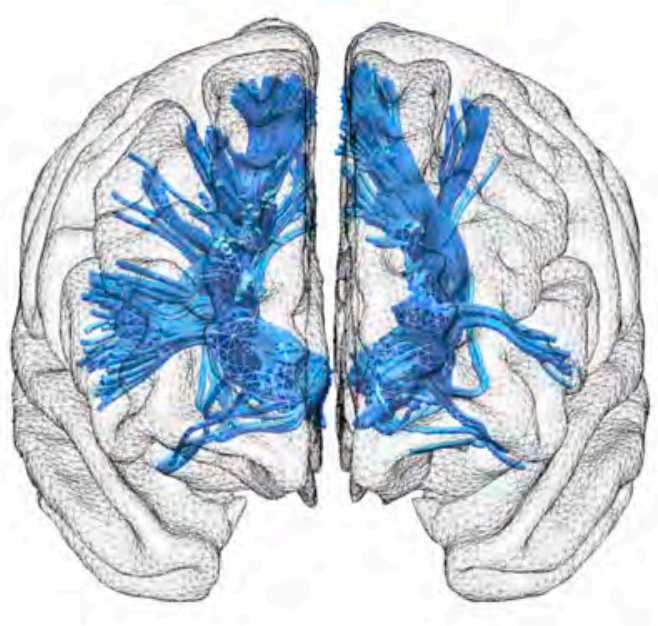

0.175 left hemisphere $\delta=-\mathbf{0 . 4 9 9} * *$

0.175 right hemisphere $\delta=-0.518 * *$
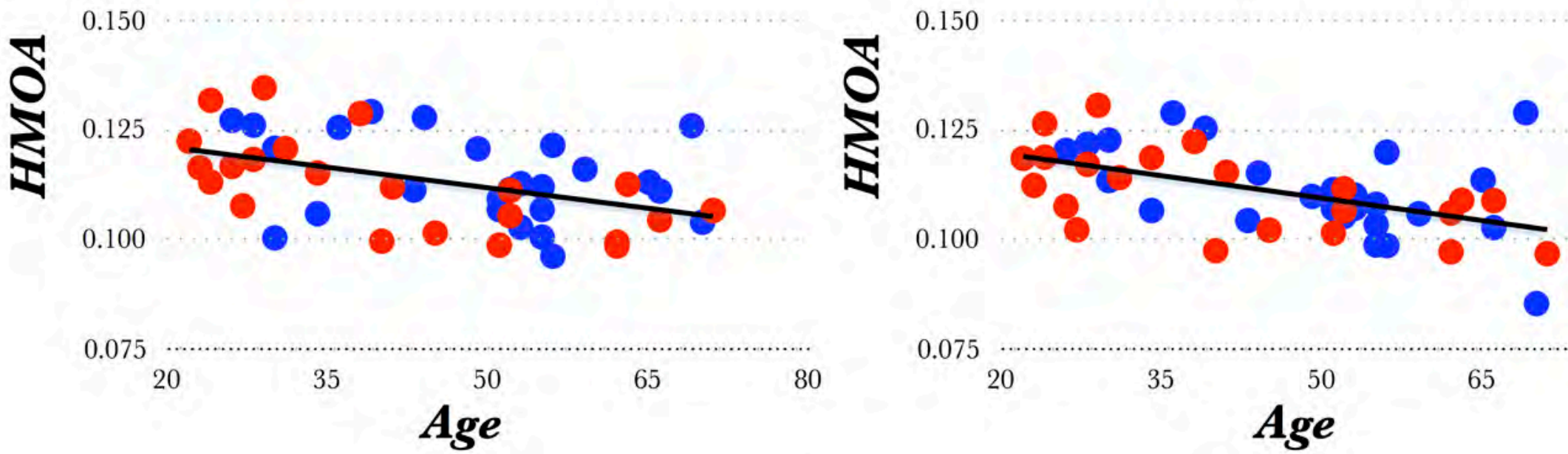

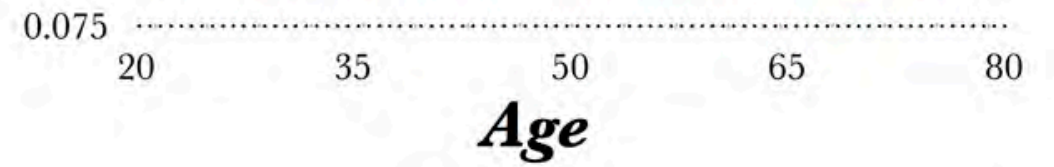


a)

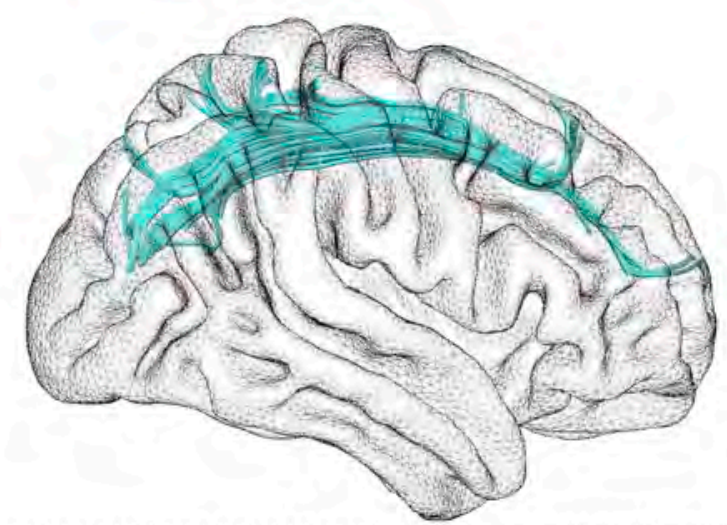

b)

$$
\delta=-0.452 *
$$
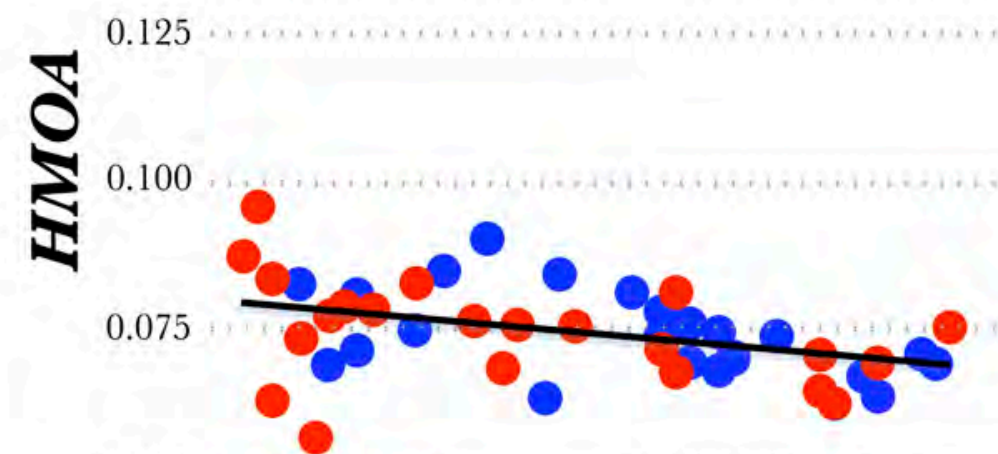

0.050

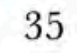

65 Age

c)

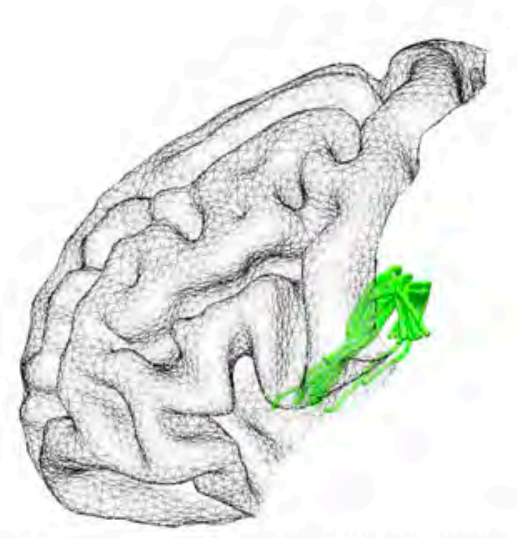

$$
\delta=-0.379 *
$$

$\underset{1}{1}$

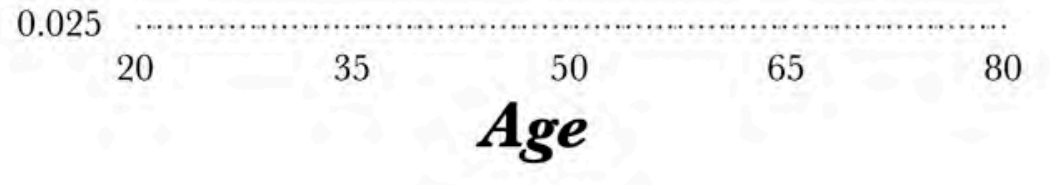

d)

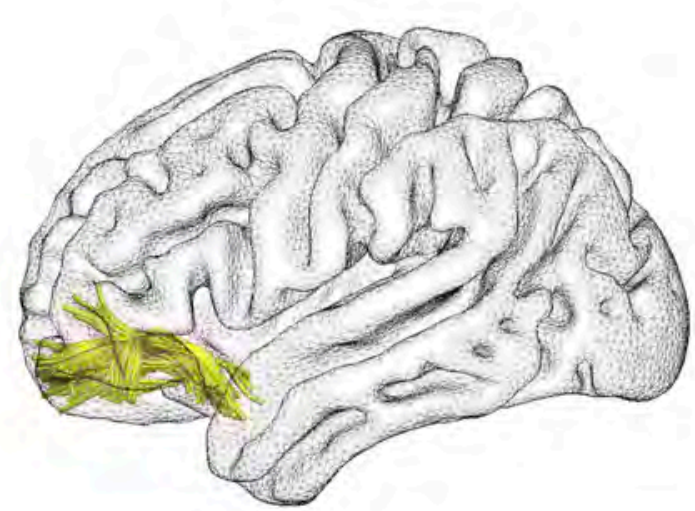

$\boldsymbol{\delta}=-0.552 * * *$

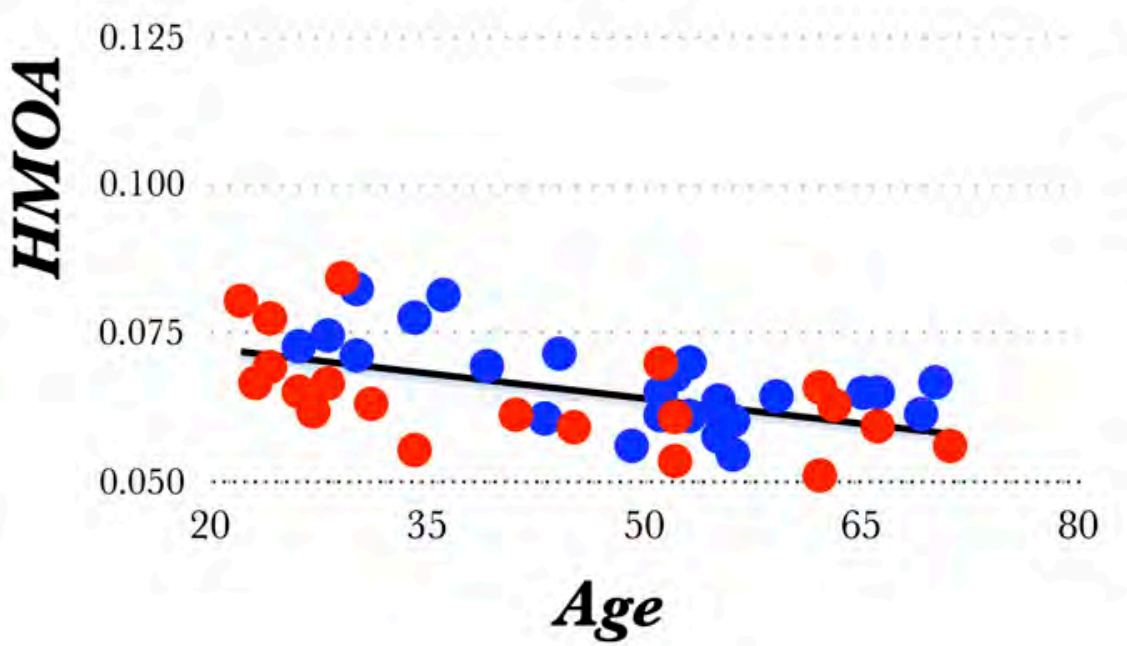

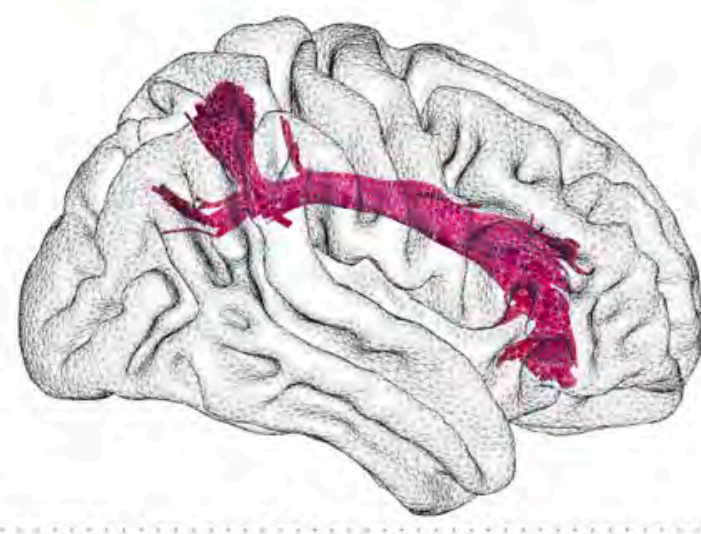

$\delta=-0.610 * * *$

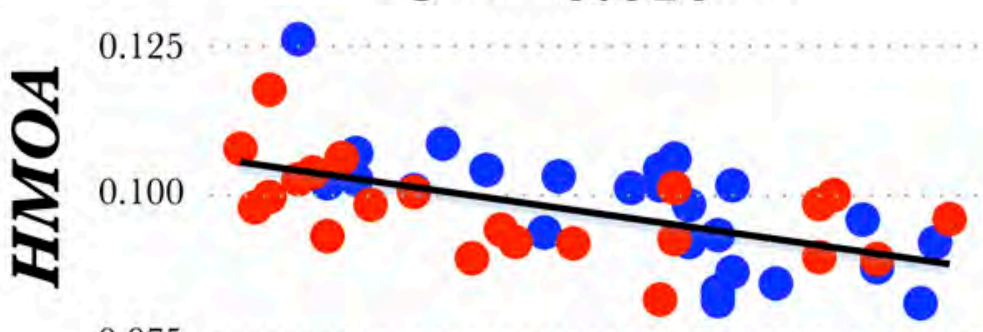

0.075

0.050

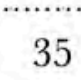

Age

e)

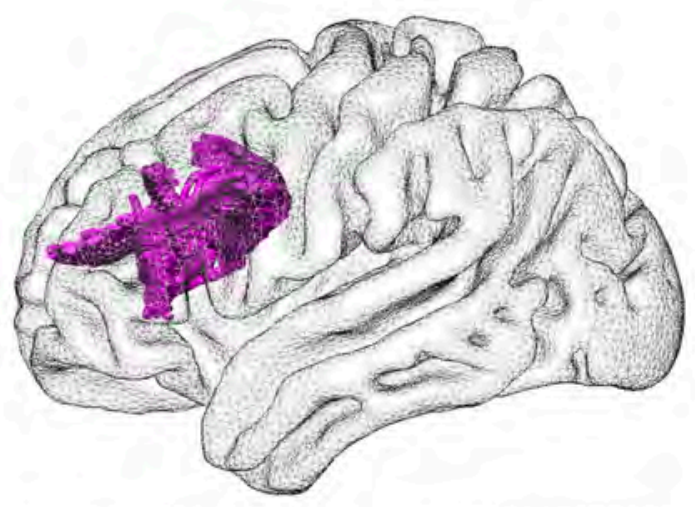

$$
\delta=-0.563 * * *
$$

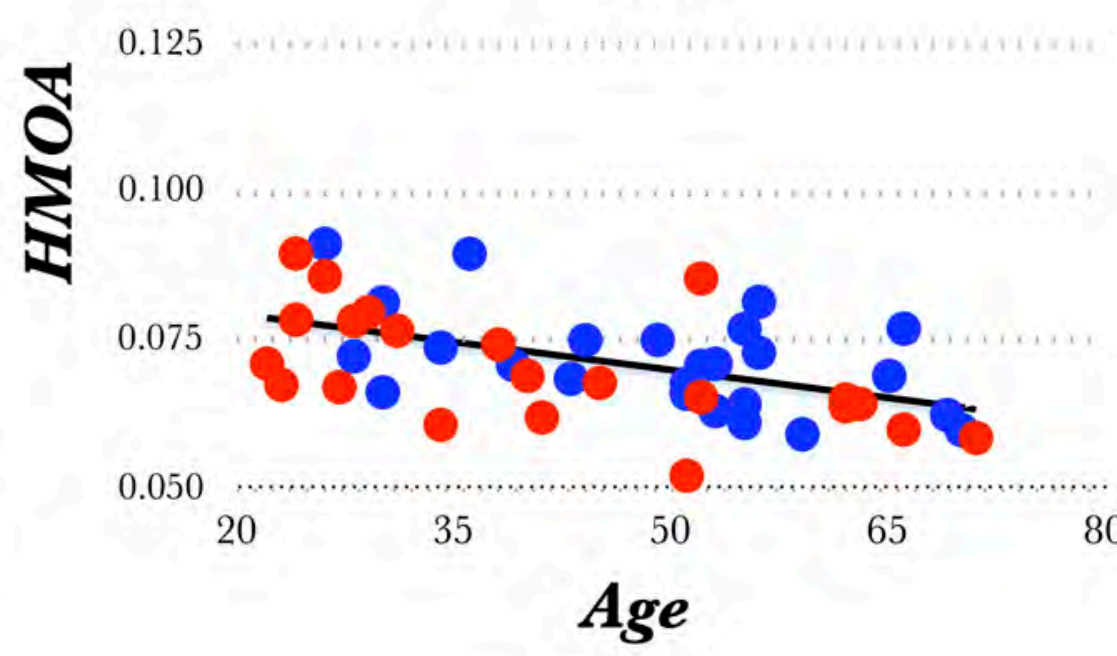




\section{SUPPLEMENTARY MATERIAL}

\section{Atlasing the frontal lobe connections and their variability due to age and education: a spherical deconvolution tractography study.}

Rojkova K. ${ }^{1,2}$, Volle E. ${ }^{1}$, Urbanski M. ${ }^{1,3}$, Humbert F. ${ }^{4}$, Dell'Acqua F. ${ }^{6}$, Thiebaut de Schotten M.*1,2,5

${ }^{1}$ CNRS UMR 7225, Inserm, UPMC-Paris6, UMR_S 1127; CRICM, GH PitiéSalpêtrière, 75013 Paris, France.

${ }^{2}$ Natbrainlab, Brain and Spine Institute, Paris, France

${ }^{3}$ Service de Médecine et de Réadaptation Gériatrique et Neurologique, Hôpitaux de Saint-Maurice, Saint-Maurice, France

${ }^{4}$ Centre de Neuroimagerie de Recherche CENIR, Groupe Hospitalier Pitié-

Salpêtrière, Paris, France

${ }^{5}$ Natbrainlab, Sackler Institute of Translational Neurodevelopment, Institute of Psychiatry, King's College London, London, UK

${ }^{6}$ Natbrainlab, Department of Neuroimaging, Institute of Psychiatry, King's College London, London, UK

*Corresponding author: michel.thiebaut@gmail.com 


\section{DIFFUSION-WEIGHTED IMAGING ACQUISITION AND PREPROCESSING}

A total of 70 near-axial slices were acquired on a Siemens 3 Tesla VERIO TIM system equipped with a 32-channel head coil. We used a fully optimised acquisition sequence for the tractography of diffusion-weighted imaging (DWI), which provided isotropic $(2 \times 2 \times 2 \mathrm{~mm})$ resolution and coverage of the whole head with a posterior-anterior phase of acquisition. The acquisition was peripherally-gated to the cardiac cycle (Conturo et al. 1995; Jones et al. 2002; Turner et al. 1990) with an echo time (TE) $=85 \mathrm{msec}$. We used a repetition time (TR) equivalent to $24 \mathrm{RR}$. At each slice location, 6 images were acquired with no diffusion gradient applied. Additionally, 60 diffusion-weighted images were acquired, in which gradient directions were uniformly distributed on the hemisphere with electrostatic repulsion. The diffusion weighting was equal to a b-value of $1500 \mathrm{sec} \mathrm{mm}^{-2}$. One supplementary image with no diffusion gradient applied but with reversed phase-encode blips was collected. This provides us with a pair of images with no diffusion gradient applied with distortions going in opposite directions. From these pairs the susceptibility-induced off-resonance field was estimated using a method similar to that described in (Andersson et al. 2003) and corrected on the whole diffusion weighted dataset using the tool TOPUP as implemented in FSL (Smith et al. 2004). Finally, at each slice, diffusionweighted data were simultaneously registered and corrected for subject motion and geometrical distortion adjusting the gradient accordingly (ExploreDTI http://www.exploredti.com) see (Leemans and Jones 2009)

\section{SPHERICAL DECONVOLUTION TRACTOGRAPHY RECONSTRUCTION}

Spherical deconvolution was chosen to estimate multiple orientations in voxels containing different populations of crossing fibers (Alexander 2006; Anderson 2005; Tournier et al. 2004). The damped version of the RichardsonLucy algorithm for spherical deconvolution (Dell'acqua et al. 2010) was calculated using a software that was developed in-house an free on demand to the authors.

Algorithm parameters were chosen as previously described (Dell'Acqua et al. 2013). A fixed-fiber response corresponding to a shape factor of $\alpha=1.5 \times 10$ $3 \mathrm{~mm} 2 / \mathrm{s}$ was chosen (Dell'Acqua et al. 2013). For each participant, a convergence speed (CS) map of the deconvolution algorithm (Dell'acqua et al. 2006) was estimated. CS map quantifies how quickly the residual fitting error between the diffusion signal, and the fiber model as identified by the deconvolution algorithm decays within each voxel. CS maps better contrast white matter regions showing a smaller partial volume effect, as compared to FA or similar anisotropy maps. Fiber orientation estimates were obtained by selecting the orientation corresponding to the peaks (local maxima) of the fiber orientation distribution (FOD) profiles. To exclude spurious local maxima, we applied both an absolute and a relative threshold on the FOD amplitude. A first "absolute" threshold was used to exclude intrinsically small local maxima due to noise or isotropic tissue. This threshold was set to 3 times the mean amplitude of a spherical FOD obtained from a gray matter isotropic voxel (and therefore also 
higher than an isotropic voxel in the cerebro-spinal fluid). A second "relative" threshold of $10 \%$ of the maximum amplitude of the FOD was applied to remove the remaining local maxima with values greater than the absolute threshold (Dell'Acqua et al. 2009).

Whole brain tractography was performed selecting every brain voxel with at least one fiber orientation as a seed voxel. From these voxels, and for each fiber orientation, streamlines were propagated using Euler integration with a step size of $1 \mathrm{~mm}$ (as described in Dell'Acqua et al. 2013). When entering a region with crossing white matter bundles, the algorithm followed the orientation vector of least curvature (as described in Schmahmann and Pandya 2007) Streamlines were halted when a voxel without fiber orientation was reached or when the curvature between two steps exceeded a threshold of $60^{\circ}$. Spherical deconvolution, fiber orientation vector estimations and tractography were performed using in-house software developed with Matlab 7.8 (http://www.mathworks.com).

\section{TRACTOGRAPHY DISSECTIONS}

We produced a CS-MNI152 template using Advanced Normalization Tools (ANTs, http://stnava.github.io/ANTs/) (Avants and Gee 2004; Avants et al. 2010), which build a template iteratively combining affine and diffeomorphic deformations (Avants et al. 2008; Klein et al. 2009). Participants were first realigned to the MNI using affine transformation. Then the template was built from all the subjects iteratively $(n=4)$ using diffeomorphic deformations.

In order to facilitate the tractography dissection, regions of interest (ROI) based on the guidelines provided in previous reports (anterior thalamic projections see (Behrens et al. 2003), main association fibers see (Catani and Thiebaut de Schotten 2008); subdivision of the arcuate see (Catani et al. 2007); fronto-pontine fibers see (Catani et al. 2008); frontal U-shape fibers see (Catani et al. 2012); fronto-striatal projections see (Cohen et al. 2009); SLF subdivision see (Thiebaut de Schotten et al. 2011a)) were defined on the CS-MNI template calculated above. In addition we performed tractography reconstruction of all the streamlines emerging from the frontal lobe and all streamlines not involving the frontal lobes. Detailed guidelines about the dissection can be found in supplementary online methods. For each participant, the CS Map was registered to the CS-MNI152 template using ANTs. The inverse deformation was then applied to the ROIs, as defined in the CS-MNI152, in order to bring them to the native space of every participant

Individual dissections of the tracts were then visually inspected and corrected in trackvis (http://www.trackvis.org), when required, by two anatomists (KR and MTS) who manually readjusted the ROIs using rigid transformation. Tract-specific measurements, such as the relative volume (volume of the tract divided by the total volume of the brain), the fractional anisotropy (mean FA value for the whole tract, Basser et al. 1994) and a new index- employed as a surrogate for tract microstructural organization (i.e. mean 
Hindrance Modulated Orientational Anisotropy or HMOA for the whole tract Dell'Acqua et al. 2013) were extracted from each dissected tract. HMOA has the advantage of providing information about the microstructural diffusion properties of distinct fiber orientations and therefore specific to the orientation of the reconstructed tracts and more accurate than classical fractional anisotropy measures, which decreases when fibers cross due to local partial volume effect.

\section{1 - Frontal and non-frontal tracts}

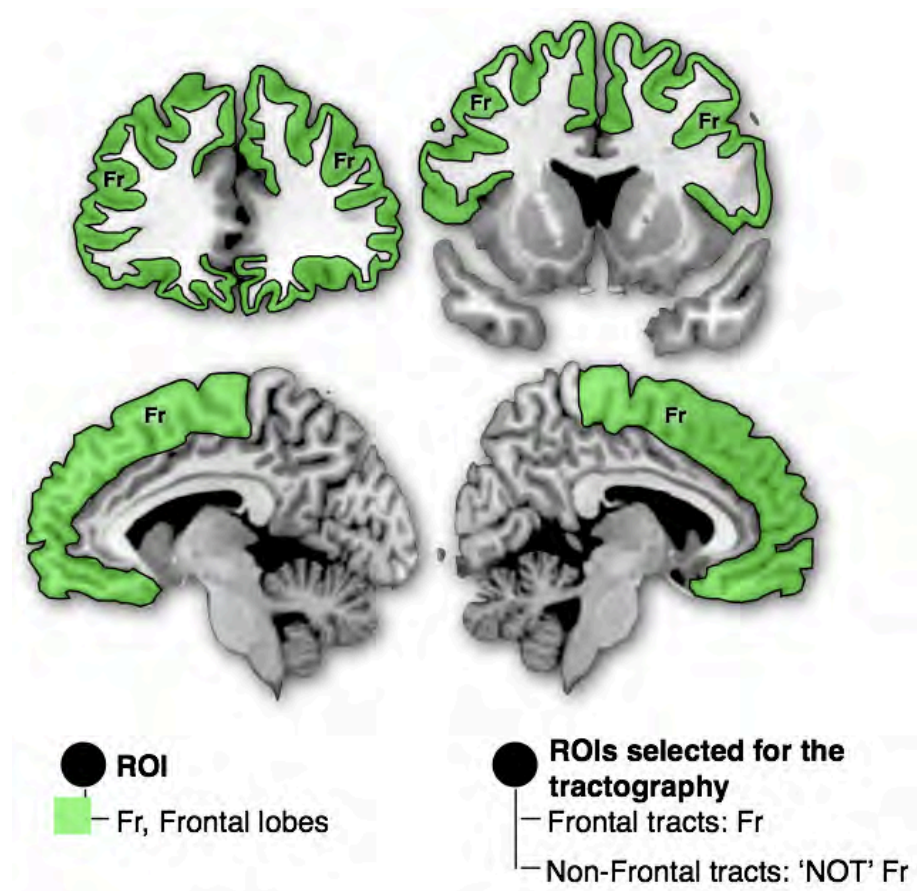

Supplementary Fig. 1: Delineation of the ROI used for the tractography of the frontal tracts and the non-frontal tracts. Left bullet, color legend for the ROI; right bullet, Boolean rules employed for the dissection.

A one-ROI approach was used to isolate the frontal lobe streamlines. We draw a region of interest including the whole frontal lobe cortical ribbon. We only displayed streamlines that were either emerging or ending inside the frontal lobe cortical ribbon.

For the non-frontal tracts we excluded from the whole brain tractography the streamlines connecting the frontal lobe cortical ribbon.

\section{2 - Superior longitudinal fasciculus}


A

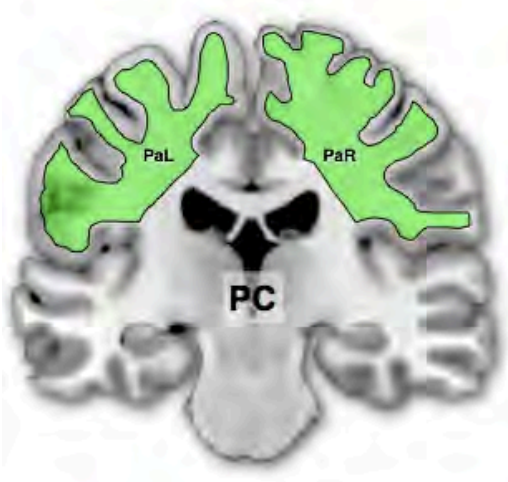

'AND' ROIS
PaL, parietal left
PaR, parietal right
SFgL, superior frontal left
- SFgR, superior frontal right
MFgL, middle frontal left
MFgR, middle frontal right
- PrgL, precentral left
PrgR, precentral right
B
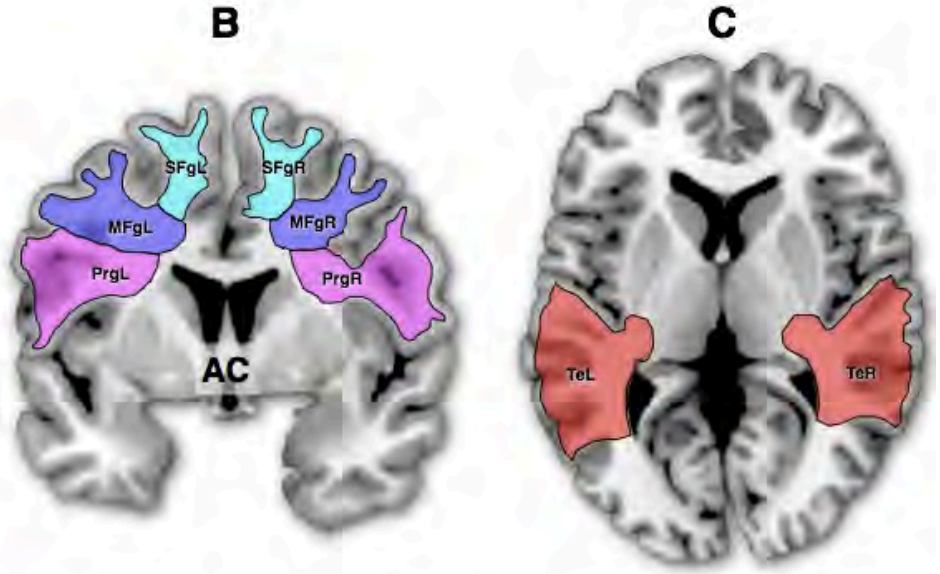

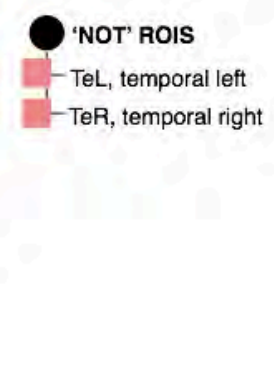

Supplementary Fig. 2: Delineation of the regions of interest (ROI) used for the tractography of the three subcomponents of the left and right parieto-frontal connections. A) Parietal ROIs in the left (PaL) and right (PaR) hemispheres. B) Frontal ROIs in the left (SFgL, MFgL and PrgL) and right (SFgR, MFgR and PrgR) hemispheres. C) Temporal ROI used to exclude the connections belonging to the temporo-frontal arcuate fasciculus in the left (TeL) and the right (TeR) hemispheres. Left bullet, color legend for the 'AND' ROIs; middle bullet, color legend for the 'NOT' ROIs; right bullet, Boolean rules employed for the dissection.

A multiple region of interests (ROIs) approach was used to isolate the three components of the superior longitudinal fasciculus (i.e. SLF I, SLF II and SLF III). Three coronal ROIs were delineated around the white matter of the superior, middle and inferior/precentral frontal gyri, and another three 'AND' ROIs were delineated posteriorly in the parietal region. Streamlines of the arcuate fasciculus projecting to the temporal lobe were excluded using an axial 'NOT' ROI in the temporal white matter (the arcuate is not part of the longitudinal system as it forms an arc to reach the temporal lobe) 


\section{3 - $\underline{\text { Cingulum }}$}
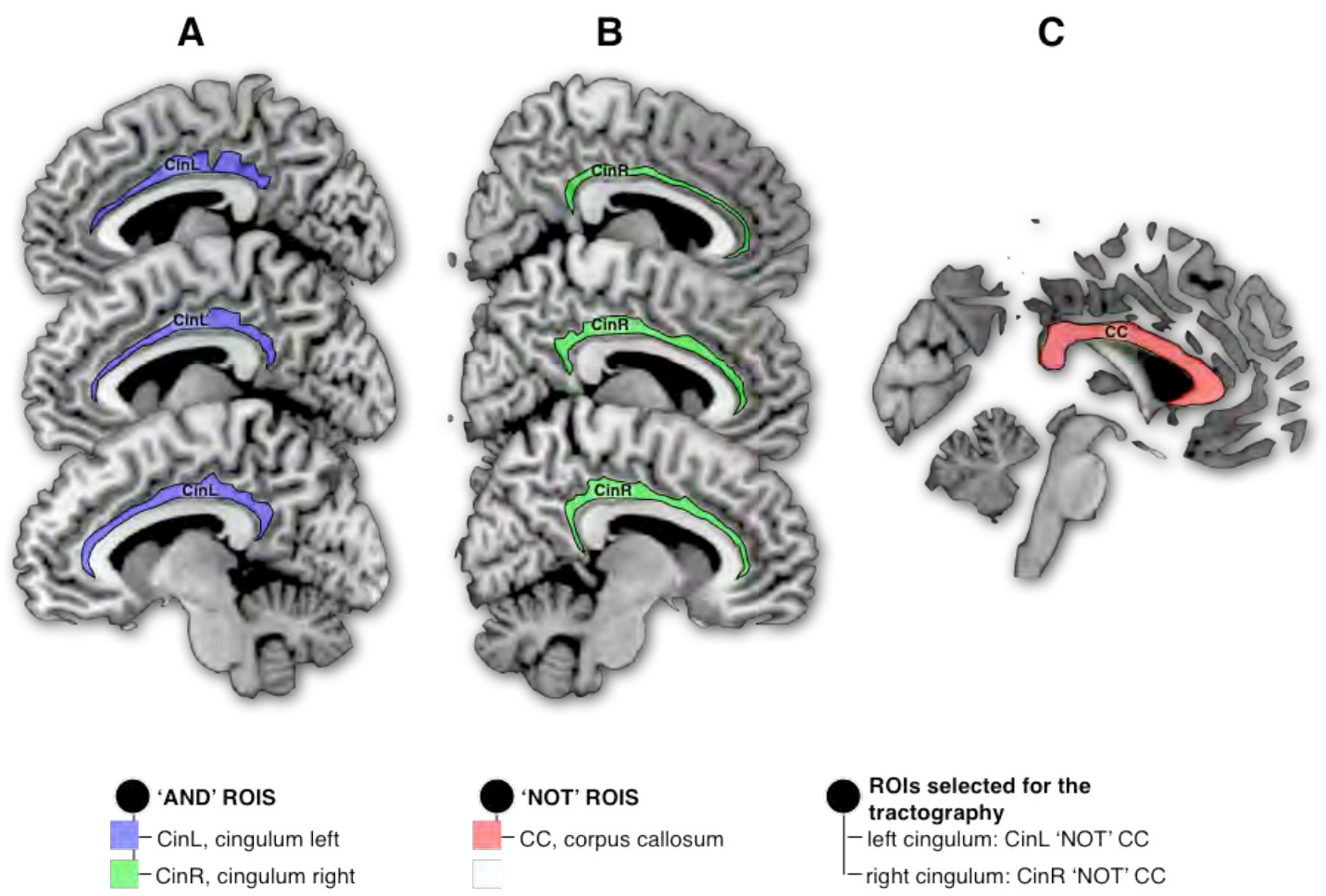

Supplementary Fig. 3: Delineation of the ROIs used for the tractography of the cingulum. A) Cingulum ROI in the left hemisphere (CinL). B) Cingulum ROI in the right hemisphere (CinR). C) Corpus callosum ROI in the medial section to exclude the connections belonging to the corpus callosum. Left bullet, color legend for the 'AND' ROIs; middle bullet, color legend for the 'NOT' ROI; right bullet, Boolean rules employed for the dissection.

A two ROIs approach was used to isolate the left and the right cingulum fasciculi. A sagittal ROI was delineated around the white matter of the cingulum for the left and the right hemispheres. Streamlines of the corpus callosum projecting to the hemisphere opposite to the ROI were excluded using a sagittal 'NOT' ROI in the corpus callosum. 


\section{4 - Uncinate and inferior fronto-occipital fasciculus (IFOF)}

A

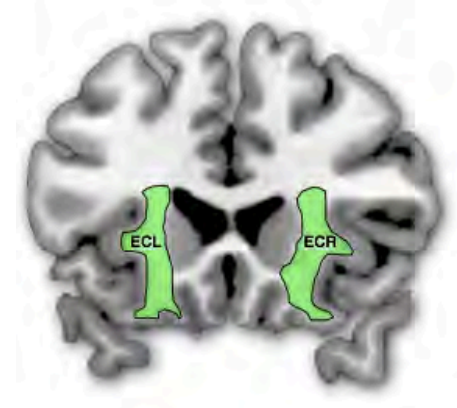

B

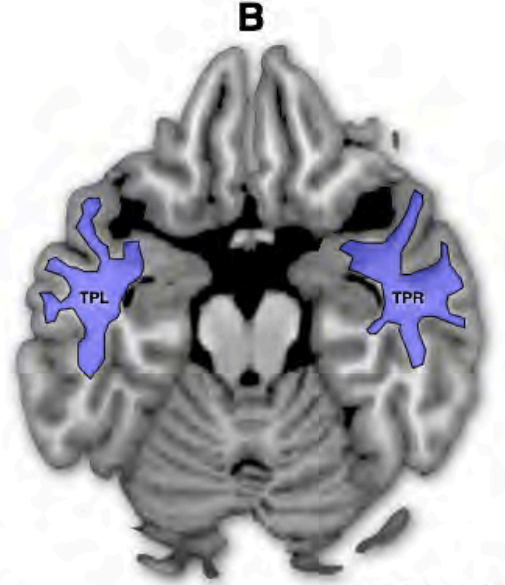

C

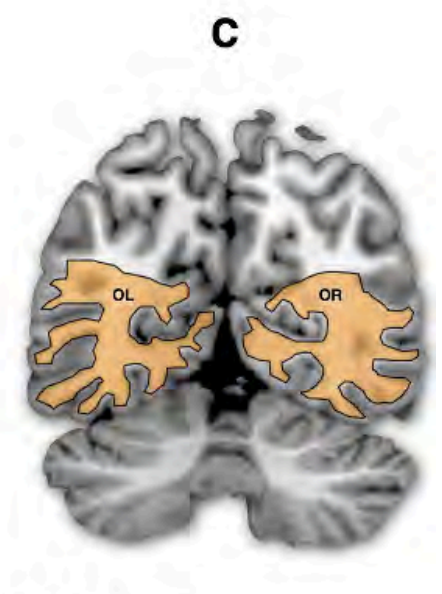

'AND' ROIS
-ECL, external/extreme capsule left
-ECR, external/extreme capsule right
-TPL, temporal left
-TPR, temporal right
-OL, occipital left
-OR, occipital right

ROls selected for the

tractography

- left uncinate: ECL 'AND' TPL

- right uncinate: ECR 'AND' TPR

- left IFOF: ECL 'AND' OL

right IFOF: ECR 'AND' OR

Supplementary Fig. 4: Delineation of the ROIs used for the tractography of the uncinate and the inferior fronto-occipital fasciculus (IFOF). A) External/Extreme capsule ROIs in the left (ECL) and the right (ECR) hemispheres. B) Temporal pole in the left (TPL) and the right (TPR) hemispheres. C) Occipital lobe in the left (OL) and the right (OR) hemispheres. Left bullet, color legend for the 'AND' ROIs; right bullet, Boolean rules employed for the dissection.

A two ROIs approach was used to isolate the left and the right uncinate and inferior fronto-occipital fasciculi. A coronal ROI was delineated around the white matter of the externe/extreme capsule, an axial 'AND' ROI in the anterior temporal region and another coronal 'AND' ROI in the occipital lobe. 


\section{5 - Anterior and long segment of the arcuate fasciculus}

A

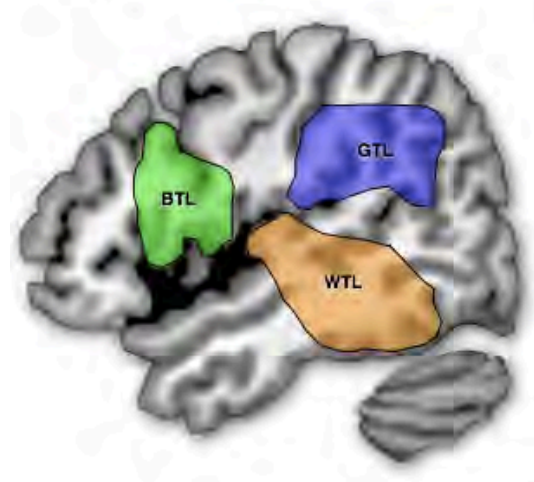

'AND' ROIS

-BTL, Broca territory left

-BTR, Broca territory right

- GTL, Geschwind territory left

- GTR, Geschwind territory right

-WTL, Wernicke territory left

-WTR, Wernicke territory right
B

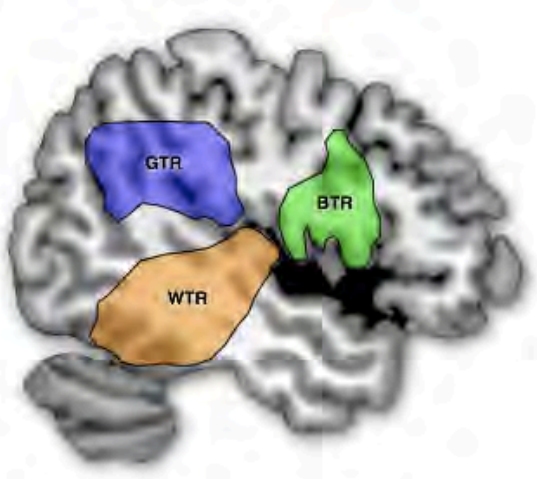

ROls selected for the

tractography

- left anterior segment: BTL 'AND' GTL

- right anterior segment: BTR 'AND' GTR

- left long segment: BTL 'AND' WTL

right long segment: BTR 'AND' WTR

Supplementary Fig. 5: Delineation of the ROIs used for the tractography of the anterior and the long segments of the left $\mathbf{A}$ ) and the right $\mathbf{B}$ ) arcuate fasciculi. Left bullet, color legend for the 'AND' ROIs; right bullet, Boolean rules employed for the dissection.

A two ROIs approach was used to isolate the left and the right anterior and long segment of the arcuate fasciculus. Three 'AND' sagittal ROIs were delineated around the white matter of the Broca territory (including ventral premotor, pars opercularis and the posterior portion of the middle frontal gyrus), Geschwind territory (including supramarginal gyrus and angular gyrus) and Wernicke territory (including posterior temporal lobe and the Heschl gyrus). 


\section{6 - Frontal corpus callosum, fronto-striatal projections, anterior thalamic radiations and fronto-pontine projections.}

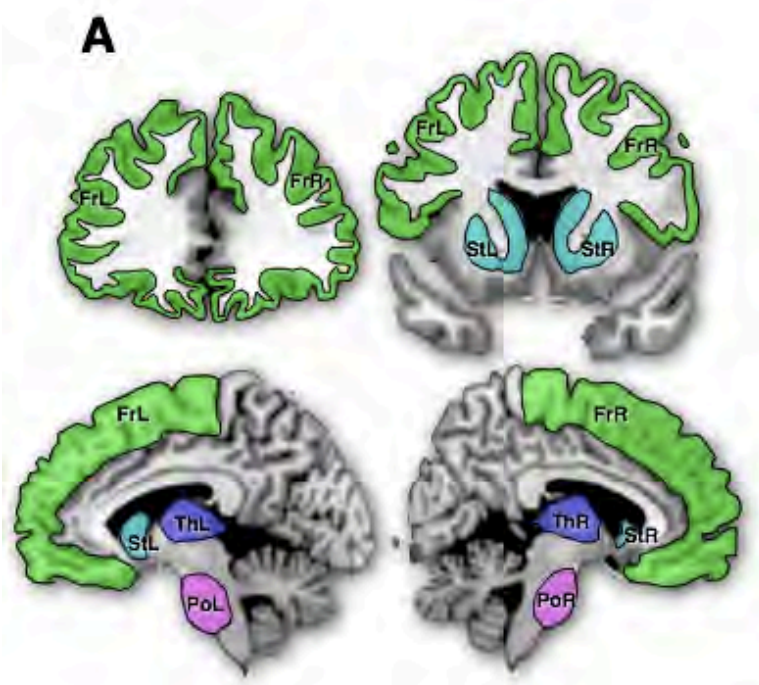

B

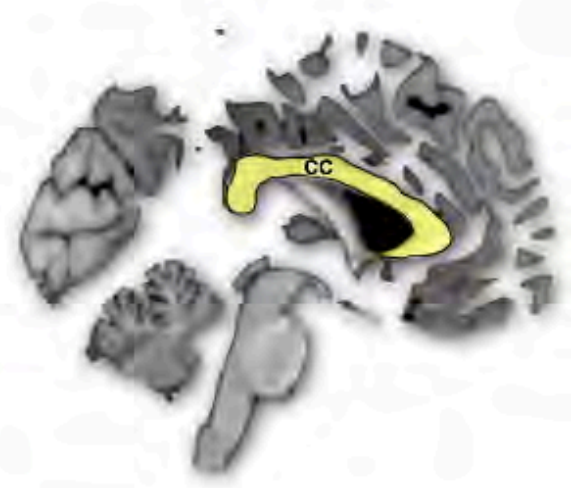

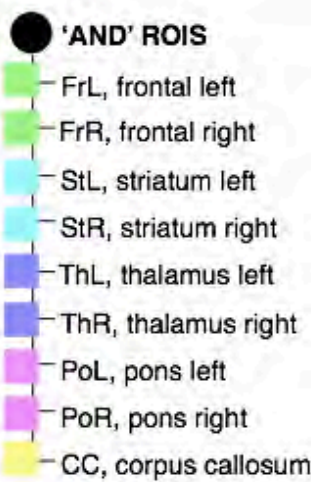

Supplementary Fig. 6: Delineation of the ROIs used for the tractography of the frontal corpus callosum, left A) and right B) fronto-striatal projections, anterior thalamic radiations and fronto-pontine projections. Left bullet, color legend for the 'AND' ROIs; right bullet, Boolean rules employed for the dissection.

A two ROIs approach was used to isolate the frontal projections of the corpus callosum and the left and right fronto-striatal, anterior thalamic radiation and fronto-pontine fibers. A 'AND' sagittal ROIs was delineated around the white matter of the corpus callosum. We also defined three 'AND' region around the whole striatum (including the caudate and the putamen), thalamus, pons whole and frontal lobe cortical ribbon and only displayed streamlines that were either emerging or ending inside these ROIs. 


\section{7 - Corticospinal tract}
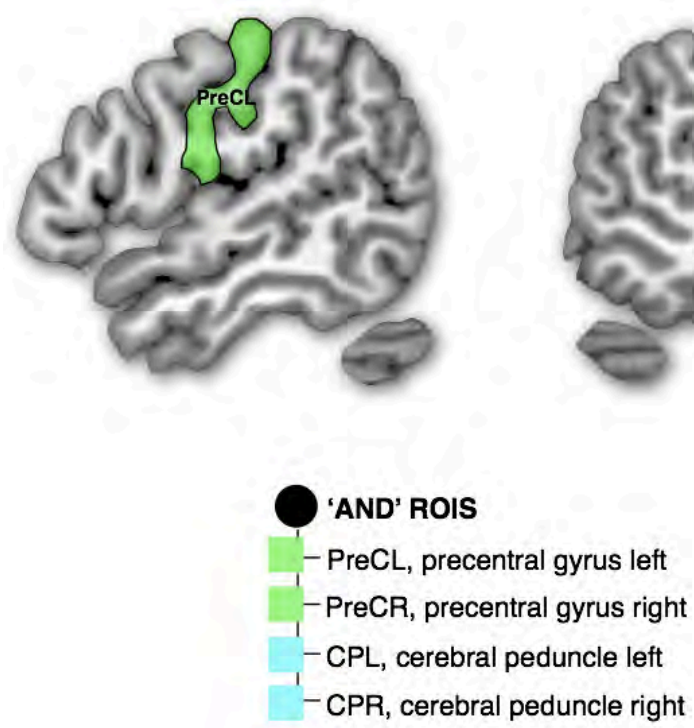
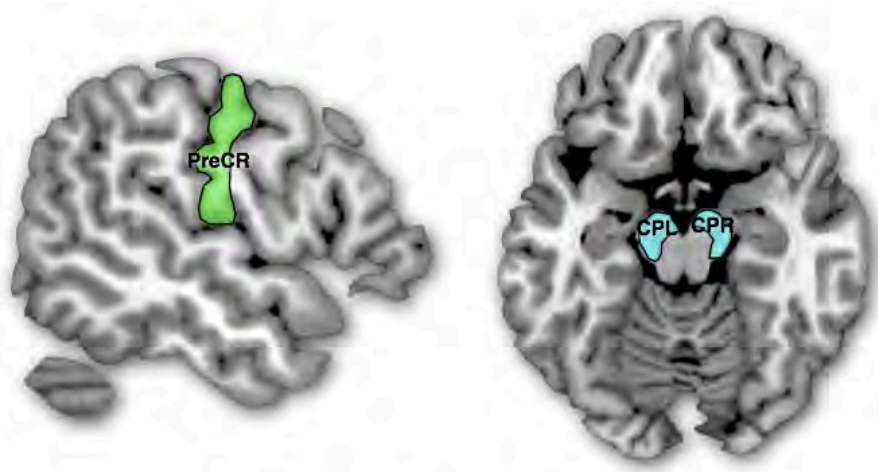

Supplementary Fig. 7: Delineation of the ROIs used for the tractography of the left and right cortico-spinal tract. Left bullet, color legend for the 'AND' ROIs; right bullet, Boolean rules employed for the dissection.

A two ROIs approach was used to isolate the cortico-spinal tract. A 'AND' ROIs was delineated around the precentral gyrus. We defined another 'AND' region around the cerebral peduncle.

\section{8 - Paracentral, hand superior, hand middle, hand superior and face U- shaped}
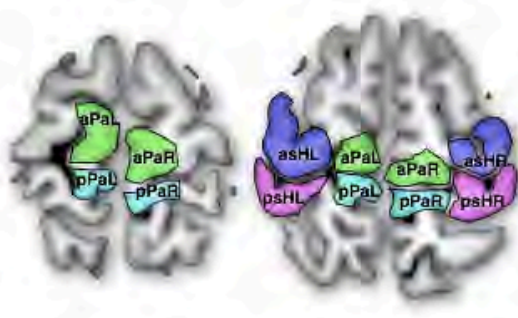

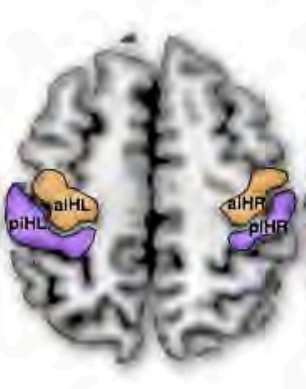

'AND' ROIS

aiHL, anterior-inferior hand left aiHR, anterior-inferior hand right piHL, posterior-inferior hand left piHR, posterior-inferior hand right -iprCL, inferior precentral left -iprCR, inferior precentral right -ipoCL, inferior postcentral left -ipoCR, inferior postcentral right

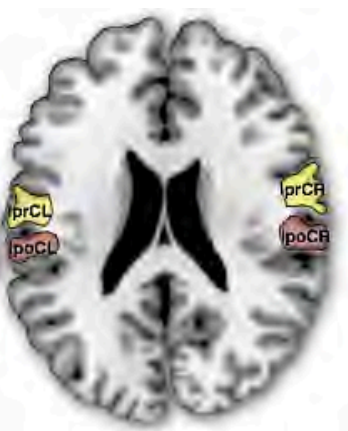

$$
\begin{aligned}
& \text { ROls selected for the } \\
& \text { tractography } \\
& \text { - left paracentral U-shaped: aPaL 'AND' pPaL } \\
& \text { - right paracentral U-shaped: aPaR 'AND' pPaR } \\
& \text { - left hand-superior U-shaped: asHL 'AND' psHL } \\
& \text { - right hand-superior U-shaped: asHR 'AND' psHR } \\
& \text { - left hand-middle U-shaped: asHL 'AND' piHL } \\
& \text { - right hand-middle U-shaped: asHR 'AND' piHR } \\
& \text { - left hand-inferior U-shaped: aiHL 'AND' piHL } \\
& \text { - right hand-inferior U-shaped: aiHR 'AND' piHR } \\
& \text { - left face U-shaped: ipreCL 'AND' ipoCL } \\
& \text { - right face U-shaped: ipreCR 'AND' ipoCR }
\end{aligned}
$$

Supplementary Fig. 8: Delineation of the ROIs used for the tractography of the left and right paracentral, hand superior, hand middle, hand inferior and face U- 
shaped. Left and middle bullets, color legend for the 'AND' ROIs; right bullet, Boolean rules employed for the dissection.

A two ROIs approach was used to isolate the paracentral, hand superior, hand middle, hand inferior and face U-shaped in the left and the right hemispheres. Eight 'AND' axial ROIs were delineated in both hemispheres around the white matter of the anterior and posterior portion of the paracentral gyrus, the superior portion of the omega sign designating the motor hand area and its corresponding post-central area, the inferior portion of the omega sign and its corresponding post-central area, the most inferior portion of the pre- and postcentral gyri. Note that each axial ROI was delineated on three consecutive slices (approximately 6 millimeters thick). An upper length threshold of 50 millimeters was applied to the dissection to discard spurious reconstructions.

\section{9 - Frontal aslant tract, superior and inferior frontal longitudinal fasciculi}
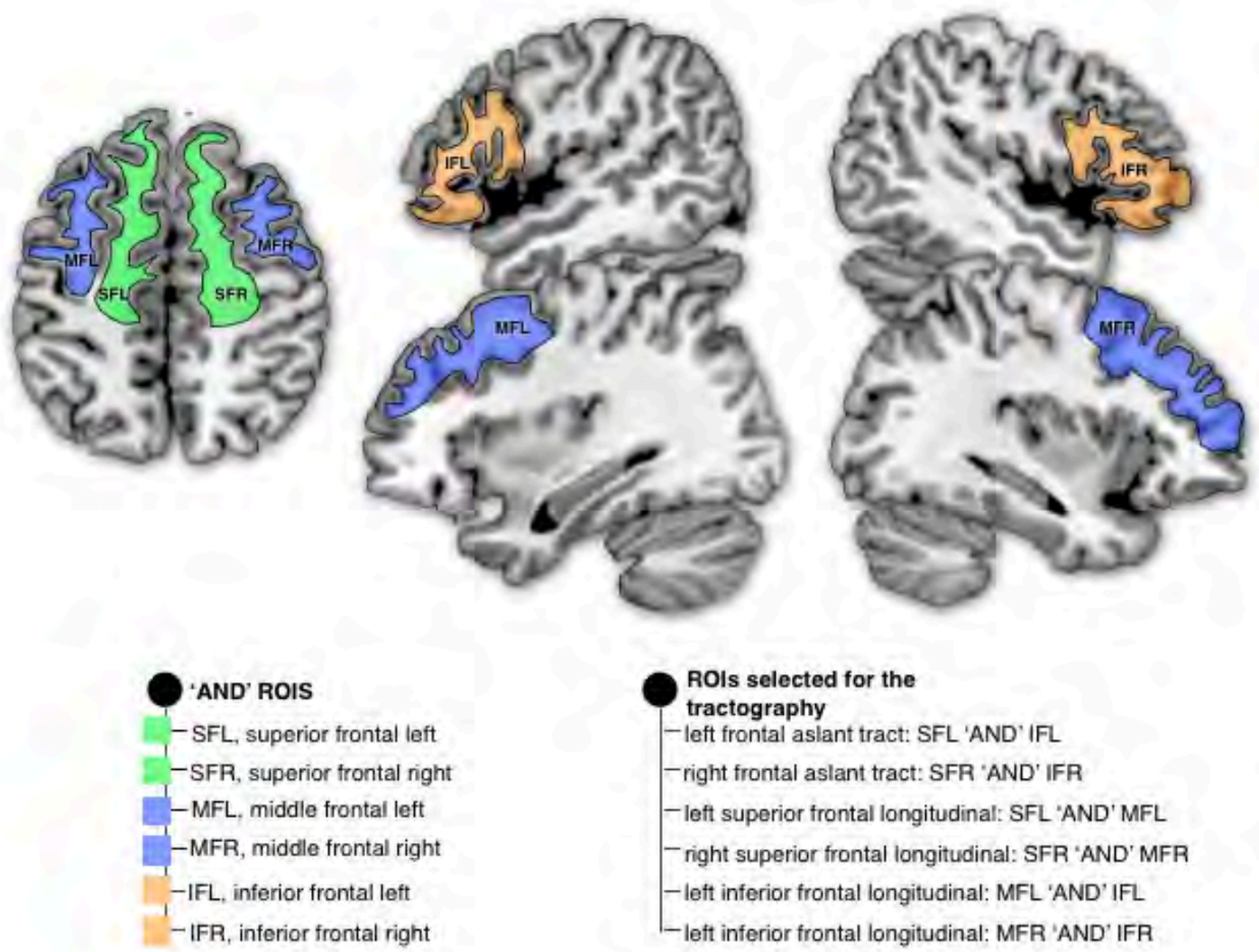

Supplementary Fig. 9: Delineation of the ROIs used for the tractography of the left and right frontal aslant tract, superior and inferior frontal longitudinal fasciculi. Left bullet, color legend for the 'AND' ROIs; right bullet, Boolean rules employed for the dissection.

A two ROIs approach was employed to isolate the left and right frontal aslant tract, superior and inferior frontal longitudinal fasciculi. For each hemisphere we delineated an axial 'AND' ROI around the white matter of the superior frontal gyrus and a sagittal 'AND' ROI around the white matter of the inferior frontal gyrus (also including the pars opercularis, triangularis and orbitalis). Finally a 
'AND' ROI was delineated around the white matter of the middle frontal gyrus in axial and sagittal sections.

\section{0 - Fronto-insular tracts}
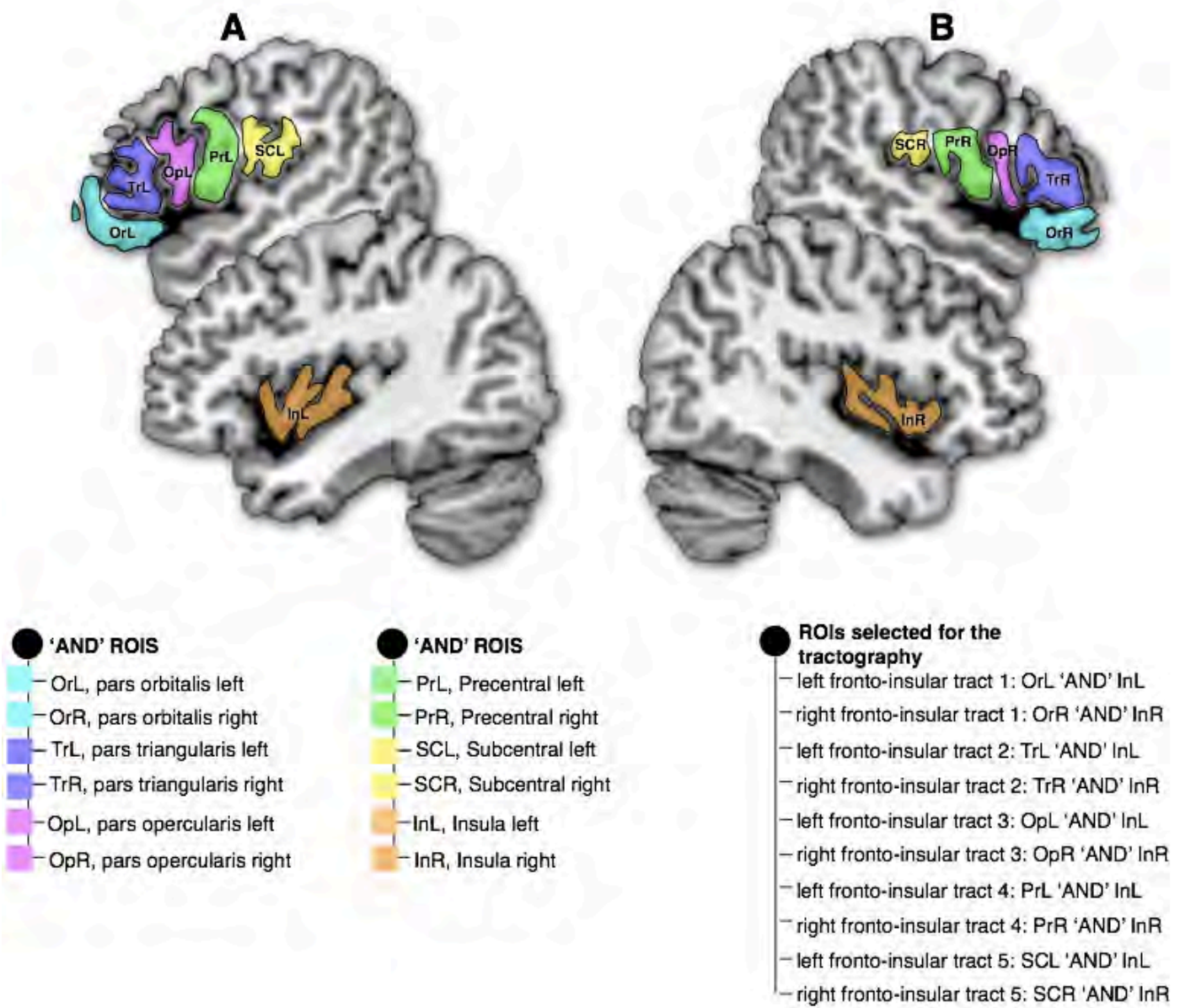

Supplementary Fig. 10: Delineation of the ROIs used for the tractography of the left and right fronto-insular tracts. Left and middle bullets, color legend for the 'AND' ROIs; right bullet, Boolean rules employed for the dissection.

A two ROIs approach was used to isolate the fronto-insular tracts. Five sagittal 'AND' ROI were delineated in both hemisphere around the white matter of the pars orbitalis, pars triangularis, pars opercularis, inferior portion of the precentral gyrus, and the subcentral gyrus. We also defined another 'AND' ROI around the whole insula displayed streamlines that were either emerging or ending inside this ROI. An upper length threshold of 50 millimeters was applied to the dissection to discard spurious reconstructions. 


\section{1 - Frontomarginal and orbitopolar tracts}
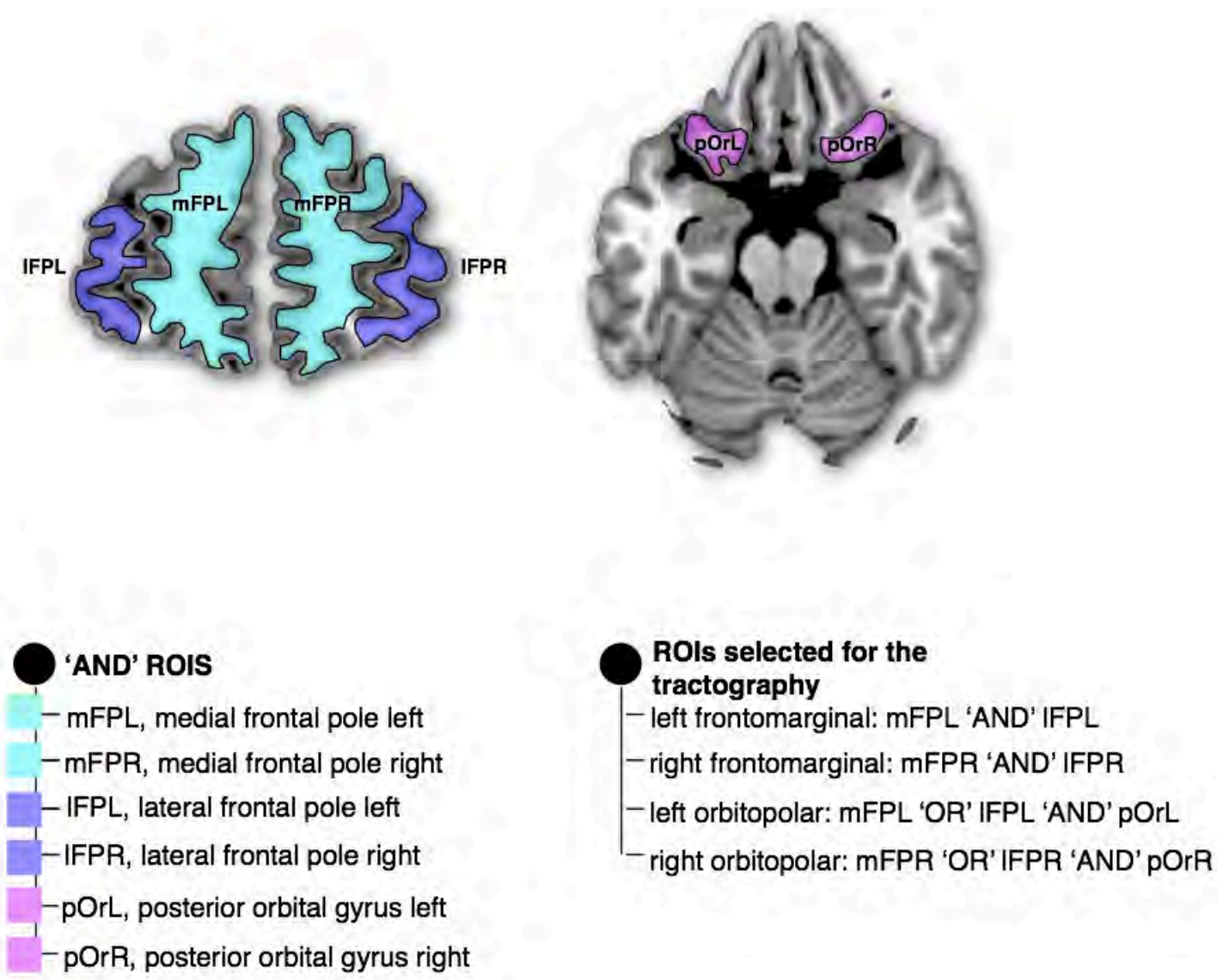

Supplementary Fig. 11: Delineation of the ROIs used for the tractography of the left and righ frontomarginal and orbitopolar and tracts. Left bullet, color legend for the 'AND' ROIs; right bullet, Boolean rules employed for the dissection.

A two-ROIs approach was used to isolate the frontal orbitopolar tract. Two coronal 'AND' ROI were delineated in both frontal lobes around the white matter of the lateral and the medial frontal pole separated by the frontomarginal sulcus, pars triangularis, pars opercularis, inferior portion of the precentral gyrus. For the orbitopolar tract we also defined another 'AND' ROI around the white matter of the posterior orbital gyrus. 


\section{MAPPING OF THE FRONTAL LOBE CONNECTIONS}

Binary individual visitation maps were created for each tract by assigning each voxel a value of 1 or 0 , depending on whether the voxel was intersected by the streamlines of the tract. Binary visitation maps of each dissected tracts were normalized to the MNI space using the same affine and diffeomorphic deformations as calculated above. We created percentage overlap maps by adding the normalized visitation maps from each subject at each point in the MNI space. Therefore, the overlap of the visitation maps varies according to intersubject variability. A similar approach has been reported in Thiebaut de Schotten et al. (Thiebaut de Schotten et al. 2011b)

3D brain rendering of the MNI152 template provided with the FSL package was calculated using MRIcroGL (http://www.cabiatl.com/mricrogl/). Percentage overlap maps of the tracts were projected onto the 3D rendering of the MNI152, in order to visualize cortical projections (Thiebaut de Schotten et al. 2011a). Probabilistic maps from the Jubrain (https://www.jubrain.fz-juelich.de) were employed to identify the corresponding Brodmann areas. The overall visualization and screenshots were performed in MRIcroGL.

\section{AgE-RELATED SHAPE CHANGES}

In this section we assessed whether the shape of the tracts change over age. We assessed this question by producing tractography maps for each decade (Supplementary Fig. 12b). We also quantified similarities using crosscorrelation between the maps for the 21-30 year-old maps and the maps for the other decades (Supplementary Fig. 12a). The result indicates that tracts reconstructed with tractography show a very high anatomical correspondence between decades and no decrease of this correspondence with age. Hence tractography in our sample did no argue for age related changes in the shape of the tract. 
a)

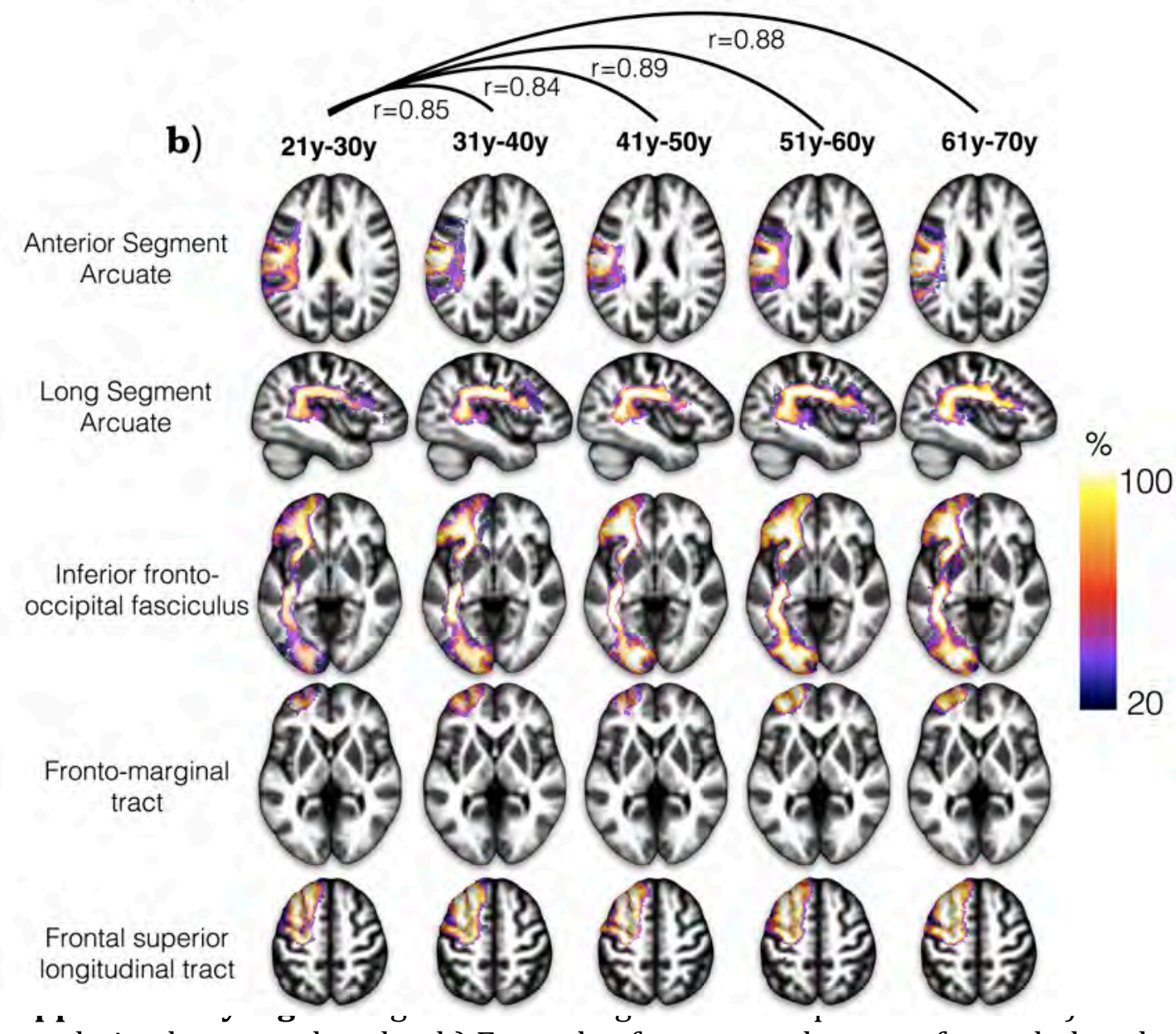

correlation between decades. b) Example of tractography maps for each decade.

\section{SUPPLEMENTARY REFERENCES}

Alexander DC (2006) An introduction to computational diffusion MRI: the diffusion tensor and beyond. In: Visualization and Processing of Tensor Fields. Springer, Berlin, pp 83-106.

Anderson AW (2005) Measurement of fiber orientation distributions using high angular resolution diffusion imaging. Magnetic resonance in medicine : official journal of the Society of Magnetic Resonance in Medicine / Society of Magnetic Resonance in Medicine 54 (5):1194-1206.

Andersson JL, Skare S, Ashburner J (2003) How to correct susceptibility distortions in spin-echo echo-planar images: application to diffusion tensor imaging. Neuroimage 20 (2):870-888.

Avants B, Gee JC (2004) Geodesic estimation for large deformation anatomical shape averaging and interpolation. Neuroimage 23 Suppl 1:S139-150.

Avants BB, Epstein CL, Grossman M, Gee JC (2008) Symmetric diffeomorphic image registration with cross-correlation: evaluating automated labeling of elderly and neurodegenerative brain. Med Image Anal 12 (1):26-41. 
Avants BB, Yushkevich P, Pluta J, Minkoff D, Korczykowski M, Detre J, Gee JC (2010) The optimal template effect in hippocampus studies of diseased populations. Neuroimage 49 (3):2457-2466.

Basser PJ, Mattiello J, Le Bihan D (1994) MR diffusion tensor spectroscopy and imaging. Biophysical Journal 66 (1):259-267.

Behrens TE, Johansen-Berg H, Woolrich MW, Smith SM, Wheeler-Kingshott CA, Boulby PA, Barker GJ, Sillery EL, Sheehan K, Ciccarelli O, Thompson AJ, Brady JM, Matthews PM (2003) Non-invasive mapping of connections between human thalamus and cortex using diffusion imaging. Nat Neurosci 6 (7):750-757.

Catani M, Allin MP, Husain M, Pugliese L, Mesulam MM, Murray RM, Jones DK (2007) Symmetries in human brain language pathways correlate with verbal recall. Proc Natl Acad Sci U S A 104 (43):17163-17168.

Catani M, Dell'acqua F, Vergani F, Malik F, Hodge H, Roy P, Valabregue R, Thiebaut de Schotten M (2012) Short frontal lobe connections of the human brain. Cortex 48 (2):273-291.

Catani M, Jones DK, Daly E, Embiricos N, Deeley Q, Pugliese L, Curran S, Robertson D, Murphy DG (2008) Altered cerebellar feedback projections in Asperger syndrome. NeuroImage 41 (4):1184-1191.

Catani M, Thiebaut de Schotten M (2008) A diffusion tensor imaging tractography atlas for virtual in vivo dissections. Cortex 44 (8):11051132.

Cohen M, Schoene-Bake J, Elger C, Weber B (2009) Connectivity-based segregation of the human striatum predicts personality characteristics. Nat Neurosci 12 (1):32-34.

Conturo TE, McKinstry RC, Aronovitz JA, Neil JJ (1995) Diffusion MRI: precision, accuracy and flow effects. NMR Biomed 8 (7-8):307-332.

Dell'Acqua F, Coward J, Simmons A, Murphy DGM, Williams S, Catani M (2009) Mapping Crossing Fibres of the Human Brain with Spherical Deconvolution: Towards an Atlas for Clinico-Anatomical Correlation Studies Proceedings of the International Society of Magnetic Resonnance Mededicine 17:3562.

Dell'acqua F, Scifo P, Rizzo G, Catani M, Simmons A, Scotti G, Fazio F (2010) A modified damped Richardson-Lucy algorithm to reduce isotropic background effects in spherical deconvolution. Neuroimage 49 (2):14461458.

Dell'acqua F, Scifo P, Rizzo G, Clark RA, Scotti G, Fazio F (2006) Convergence Maps from Richardson-Lucy Spherical Deconvolution Algorithm for the Detection of White Matter in HARDI. NeuroImage 31 (S1):S953-S953.

Dell'Acqua F, Simmons A, Williams SC, Catani M (2013) Can spherical deconvolution provide more information than fiber orientations? Hindrance modulated orientational anisotropy, a true-tract specific index to characterize white matter diffusion. Hum Brain Mapp 34 (10):24642483.

Jones DK, Griffin LD, Alexander DC, Catani M, Horsfield MA, Howard RJ, Williams SC (2002) Spatial normalization and averaging of diffusion tensor MRI data sets. NeuroImage 17 (2):592-617.

Klein A, Andersson J, Ardekani BA, Ashburner J, Avants B, Chiang MC, Christensen GE, Collins DL, Gee J, Hellier P, Song JH, Jenkinson M, Lepage 
C, Rueckert D, Thompson P, Vercauteren T, Woods RP, Mann JJ, Parsey RV (2009) Evaluation of 14 nonlinear deformation algorithms applied to human brain MRI registration. NeuroImage 46 (3):786-802.

Leemans A, Jones DK (2009) The B-matrix must be rotated when correcting for subject motion in DTI data. Magnetic Resonance in Medecine 61 (6):13361349.

Schmahmann JD, Pandya DN (2007) The complex history of the fronto-occipital fasciculus. Journal of the History of the Neurosciences 16 (4):362-377.

Smith SM, Jenkinson M, Woolrich MW, Beckmann CF, Behrens TEJ, JohansenBerg H, Bannister PR, De Luca M, Drobnjak I, Flitney DE, Niazy RK, Saunders J, Vickers J, Zhang Y, De Stefano N, Brady JM, Matthews PM (2004) Advances in functional and structural MR image analysis and implementation as FSL. Neuroimage 23 Suppl 1:S208-219.

Thiebaut de Schotten M, Dell'Acqua F, Forkel SJ, Simmons A, Vergani F, Murphy DG, Catani M (2011a) A lateralized brain network for visuospatial attention. Nat Neurosci 14 (10):1245-1246.

Thiebaut de Schotten M, Ffytche DH, Bizzi A, Dell'Acqua F, Allin M, Walshe M, Murray R, Williams SC, Murphy DG, Catani M (2011b) Atlasing location, asymmetry and inter-subject variability of white matter tracts in the human brain with MR diffusion tractography. Neuroimage 54 (1):49-59.

Tournier JD, Calamante F, Gadian DG, Connelly A (2004) Direct estimation of the fiber orientation density function from diffusion-weighted MRI data using spherical deconvolution. NeuroImage 23 (3):1176-1185.

Turner R, Le Bihan D, Maier J, Vavrek R, Hedges LK, Pekar J (1990) Echo-planar imaging of intravoxel incoherent motion. Radiology 177 (2):407-414. 
Tract specific measurements

\begin{tabular}{|c|c|c|c|c|c|}
\hline Tracts & $\begin{array}{l}\text { Success } \\
\text { rate }\end{array}$ & Volume (cm3) & Volume (corrected) & FA & HMOA \\
\hline SLF1 L & $100 \%$ & $6.02 \pm 2.82$ & $0.0041 \pm 0.0020$ & $0.223 \pm 0.040$ & $301.95 \pm 35.60$ \\
\hline SLF1 R & $98 \%$ & $5.64 \pm 3.50$ & $0.0038 \pm 0.0023$ & $0.194 \pm 0.045$ & $296.85 \pm 31.71$ \\
\hline SLF2 L & $100 \%$ & $25.24 \pm 8.16$ & $0.0172 \pm 0.0055$ & $0.152 \pm 0.018$ & $355.48 \pm 33.92$ \\
\hline SLF2 R & $100 \%$ & $25.65 \pm 6.30$ & $0.0175 \pm 0.0043$ & $0.168 \pm 0.013$ & $390.20 \pm 39.23$ \\
\hline SLF3 L & $100 \%$ & $15.77 \pm 6.04$ & $0.0108 \pm 0.0041$ & $0.181 \pm 0.024$ & $340.26 \pm 38.73$ \\
\hline SLF3 R & $100 \%$ & $25.85 \pm 6.99$ & $0.0176 \pm 0.0045$ & $0.196 \pm 0.017$ & $388.41 \pm 36.16$ \\
\hline Cingulum L & $100 \%$ & $42.89 \pm 7.73$ & $0.0293 \pm 0.0053$ & $0.313 \pm 0.018$ & $439.84 \pm 45.73$ \\
\hline Cingulum R & $100 \%$ & $39.47 \pm 7.24$ & $0.0269 \pm 0.0048$ & $0.270 \pm 0.023$ & $405.62 \pm 44.44$ \\
\hline Uncinate L & $100 \%$ & $8.36 \pm 2.95$ & $0.0057 \pm 0.0020$ & $0.157 \pm 0.014$ & $337.61 \pm 34.07$ \\
\hline Uncinate $\mathbf{R}$ & $100 \%$ & $7.18 \pm 3.12$ & $0.0049 \pm 0.0021$ & $0.165 \pm 0.016$ & $343.31 \pm 35.20$ \\
\hline Arcuate long segment $L$ & $94 \%$ & $13.01 \pm 7.18$ & $0.0089 \pm 0.0048$ & $0.123 \pm 0.013$ & $433.68 \pm 54.68$ \\
\hline Arcuate long segment $R$ & $100 \%$ & $8.60 \pm 4.84$ & $0.0060 \pm 0.0035$ & $0.156 \pm 0.025$ & $412.57 \pm 38.00$ \\
\hline Arcuate anterior segment $L$ & $100 \%$ & $7.69 \pm 2.68$ & $0.0052 \pm 0.0018$ & $0.118 \pm 0.012$ & $309.40 \pm 46.92$ \\
\hline Arcuate anterior segment $R$ & $100 \%$ & $14.42 \pm 4.28$ & $0.0099 \pm 0.0030$ & $0.169 \pm 0.019$ & $392.35 \pm 40.07$ \\
\hline Inferior fronto occipital fasciculus $L$ & $100 \%$ & $22.29 \pm 6.88$ & $0.0152 \pm 0.0045$ & $0.144 \pm 0.012$ & $475.52 \pm 48.74$ \\
\hline Inferior fronto occipital fasciculus $R$ & $100 \%$ & $22.35 \pm 8.21$ & $0.0153 \pm 0.0055$ & $0.150 \pm 0.013$ & $476.51 \pm 51.94$ \\
\hline Corpus callosum & $100 \%$ & $128.30 \pm 27.76$ & $0.0875 \pm 0.0177$ & $0.328 \pm 0.015$ & $478.34 \pm 52.28$ \\
\hline Cortico-spinal L & $100 \%$ & $17.89 \pm 5.12$ & $0.0122 \pm 0.0034$ & $0.206 \pm 0.026$ & $517.99 \pm 50.46$ \\
\hline Cortico-spinal R & $100 \%$ & $19.63 \pm 5.11$ & $0.0134 \pm 0.0033$ & $0.215 \pm 0.028$ & $507.66 \pm 46.13$ \\
\hline Anterior thalamic radiations $L$ & $100 \%$ & $43.53 \pm 32.64$ & $0.0295 \pm 0.0216$ & $0.166 \pm 0.015$ & $452.72 \pm 40.62$ \\
\hline Anterior thalamic radiations $R$ & $100 \%$ & $46.29 \pm 35.96$ & $0.0315 \pm 0.0238$ & $0.158 \pm 0.018$ & $443.75 \pm 39.44$ \\
\hline Fronto-striatal L & $100 \%$ & $38.68 \pm 7.64$ & $0.0263 \pm 0.0046$ & $0.160 \pm 0.010$ & $320.34 \pm 29.51$ \\
\hline Fronto-striatal R & $100 \%$ & $35.61 \pm 7.72$ & $0.0243 \pm 0.0050$ & $0.168 \pm 0.013$ & $322.25 \pm 33.97$ \\
\hline Fronto pontine L & $100 \%$ & $19.60 \pm 5.64$ & $0.0134 \pm 0.0037$ & $0.387 \pm 0.021$ & $461.17 \pm 44.39$ \\
\hline Fronto pontine $\mathbf{R}$ & $100 \%$ & $17.77 \pm 5.73$ & $0.0121 \pm 0.0038$ & $0.405 \pm 0.018$ & $568.68 \pm 52.86$ \\
\hline Paracentral $\mathrm{U}$ tract $\mathrm{L}$ & $68 \%$ & $0.61 \pm 0.45$ & $0.0004 \pm 0.0003$ & $0.141 \pm 0.044$ & $211.93 \pm 48.09$ \\
\hline Paracentral $U$ tract $R$ & $66 \%$ & $0.50 \pm 0.40$ & $0.0003 \pm 0.0003$ & $0.108 \pm 0.041$ & $215.78 \pm 50.88$ \\
\hline HandSup U tract L & $100 \%$ & $3.25 \pm 1.43$ & $0.0022 \pm 0.0009$ & $0.126 \pm 0.032$ & $344.69 \pm 37.52$ \\
\hline HandSup U tract R & $98 \%$ & $2.63 \pm 1.10$ & $0.0018 \pm 0.0007$ & $0.142 \pm 0.031$ & $341.65 \pm 34.89$ \\
\hline Handmid U tract $L$ & $38 \%$ & $1.19 \pm 1.15$ & $0.0008 \pm 0.0007$ & $0.086 \pm 0.025$ & $301.86 \pm 41.54$ \\
\hline Handmid U tract $R$ & $100 \%$ & $1.40 \pm 0.86$ & $0.0010 \pm 0.0006$ & $0.146 \pm 0.030$ & $308.32 \pm 31.49$ \\
\hline Handinf $U$ tract $L$ & $94 \%$ & $3.40 \pm 1.58$ & $0.0023 \pm 0.0011$ & $0.126 \pm 0.024$ & $296.47 \pm 35.34$ \\
\hline Handinf $\mathrm{U}$ tract $\mathrm{R}$ & $100 \%$ & $3.07 \pm 1.32$ & $0.0021 \pm 0.0009$ & $0.162 \pm 0.027$ & $312.03 \pm 34.67$ \\
\hline Face $U$ tract $L$ & $91 \%$ & $1.36 \pm 0.97$ & $0.0009 \pm 0.0007$ & $0.116 \pm 0.021$ & $229.72 \pm 39.65$ \\
\hline Face $U$ tract $R$ & $87 \%$ & $1.17 \pm 0.67$ & $0.0008 \pm 0.0005$ & $0.180 \pm 0.029$ & $229.20 \pm 44.23$ \\
\hline Frontal aslant Tract $L$ & $100 \%$ & $16.62 \pm 4.54$ & $0.0113 \pm 0.0029$ & $0.120 \pm 0.008$ & $369.19 \pm 40.78$ \\
\hline Frontal aslant Tract $R$ & $100 \%$ & $16.30 \pm 5.01$ & $0.0111 \pm 0.0033$ & $0.143 \pm 0.011$ & $363.02 \pm 28.74$ \\
\hline Fronto Insular tract $1 \mathrm{~L}$ & $26 \%$ & $0.40 \pm 0.20$ & $0.0003 \pm 0.0001$ & $0.135 \pm 0.032$ & $257.63 \pm 58.76$ \\
\hline Fronto Insular tract $1 \mathrm{R}$ & $74 \%$ & $0.59 \pm 0.43$ & $0.0004 \pm 0.0003$ & $0.217 \pm 0.058$ & $241.77 \pm 35.74$ \\
\hline Fronto Insular tract $2 \mathrm{~L}$ & $42 \%$ & $0.52 \pm 0.26$ & $0.0004 \pm 0.0002$ & $0.109 \pm 0.020$ & $287.52 \pm 46.22$ \\
\hline Fronto Insular tract 2 R & $85 \%$ & $1.32 \pm 0.69$ & $0.0009 \pm 0.0005$ & $0.154 \pm 0.029$ & $253.80 \pm 32.35$ \\
\hline Fronto Insular tract $3 \mathrm{~L}$ & $96 \%$ & $1.30 \pm 0.80$ & $0.0009 \pm 0.0006$ & $0.160 \pm 0.030$ & $239.89 \pm 36.03$ \\
\hline Fronto Insular tract $3 \mathbf{R}$ & $100 \%$ & $3.24 \pm 1.09$ & $0.0022 \pm 0.0008$ & $0.177 \pm 0.019$ & $241.02 \pm 25.01$ \\
\hline Fronto Insular tract $4 \mathrm{~L}$ & $100 \%$ & $2.17 \pm 0.87$ & $0.0015 \pm 0.0006$ & $0.146 \pm 0.020$ & $223.84 \pm 19.55$ \\
\hline Fronto Insular tract $4 \mathrm{R}$ & $100 \%$ & $2.78 \pm 0.86$ & $0.0019 \pm 0.0006$ & $0.167 \pm 0.019$ & $220.67 \pm 17.80$ \\
\hline Fronto Insular tract $5 \mathrm{~L}$ & $98 \%$ & $1.59 \pm 0.64$ & $0.0011 \pm 0.0005$ & $0.148 \pm 0.024$ & $213.80 \pm 18.14$ \\
\hline Fronto Insular tract $5 \mathrm{R}$ & $100 \%$ & $2.96 \pm 0.89$ & $0.0020 \pm 0.0006$ & $0.187 \pm 0.022$ & $214.64 \pm 19.99$ \\
\hline Frontal superior longitudinal L & $100 \%$ & $12.72 \pm 3.89$ & $0.0087 \pm 0.0026$ & $0.128 \pm 0.011$ & $320.68 \pm 21.81$ \\
\hline Frontal superior longitudinal $\mathbf{R}$ & $100 \%$ & $12.08 \pm 4.08$ & $0.0082 \pm 0.0027$ & $0.158 \pm 0.015$ & $311.49 \pm 28.36$ \\
\hline Frontal inferior longitudinal L & $100 \%$ & $3.96 \pm 2.21$ & $0.0027 \pm 0.0015$ & $0.146 \pm 0.024$ & $283.81 \pm 36.17$ \\
\hline Frontal inferior longitudinal R & $100 \%$ & $6.82 \pm 3.03$ & $0.0047 \pm 0.0020$ & $0.188 \pm 0.027$ & $288.05 \pm 34.63$ \\
\hline Frontal orbito polar $\mathrm{L}$ & $98 \%$ & $2.92 \pm 1.46$ & $0.0020 \pm 0.0010$ & $0.141 \pm 0.017$ & $260.24 \pm 33.69$ \\
\hline Frontal orbito polar $\mathbf{R}$ & $100 \%$ & $3.27 \pm 1.68$ & $0.0022 \pm 0.0011$ & $0.168 \pm 0.025$ & $254.76 \pm 33.94$ \\
\hline Fronto Marginal tract $L$ & $91 \%$ & $2.65 \pm 1.43$ & $0.0018 \pm 0.0009$ & $0.131 \pm 0.019$ & $236.93 \pm 30.88$ \\
\hline Fronto Marginal tract $\mathbf{R}$ & $100 \%$ & $4.17 \pm 1.91$ & $0.0028 \pm 0.0012$ & $0.170 \pm 0.018$ & $231.25 \pm 30.76$ \\
\hline
\end{tabular}


Statistics

\begin{tabular}{|c|c|c|c|c|c|c|}
\hline Tracts & Age (Vol) & $\begin{array}{l}\text { Age } \\
\text { (FA) }\end{array}$ & $\begin{array}{c}\text { Age } \\
\text { (HMOA) }\end{array}$ & $\begin{array}{l}\text { Education } \\
\text { (Vol) }\end{array}$ & $\begin{array}{l}\text { Education } \\
\text { (FA) }\end{array}$ & $\begin{array}{c}\text { Education } \\
\text { (HMOA) }\end{array}$ \\
\hline SLF1 L & 0.053 & 0.565 & 0.728 & 0.894 & 0.291 & 0.986 \\
\hline SLF1 R & 0.094 & 0.548 & 0.002 & 0.482 & 0.306 & 0.862 \\
\hline SLF2 L & 0.485 & 0.871 & 0.136 & 0.805 & 0.226 & 0.323 \\
\hline SLF2 R & 0.965 & 0.845 & 0.244 & 0.713 & 0.759 & 0.946 \\
\hline SLF3 L & 0.354 & 0.579 & 0.033 & 0.529 & 0.908 & 0.824 \\
\hline SLF3 R & 0.174 & 0.844 & 0.000 & 0.765 & 0.623 & 0.229 \\
\hline Cingulum L & 0.907 & 0.020 & 0.016 & 0.397 & 0.806 & 0.828 \\
\hline Cingulum $\mathbf{R}$ & 0.807 & 0.370 & 0.558 & 0.678 & 0.928 & 0.793 \\
\hline Uncinate L & 0.268 & 0.334 & 0.767 & 0.086 & 0.843 & 0.721 \\
\hline Uncinate R & 0.973 & 0.016 & 0.028 & 0.265 & 0.172 & 0.206 \\
\hline Arcuate long segment $L$ & 0.385 & 0.006 & 0.152 & 0.498 & 0.528 & 0.414 \\
\hline Arcuate long segment $R$ & 0.295 & 0.694 & 0.201 & 0.934 & 0.638 & 0.737 \\
\hline Arcuate anterior segment $L$ & 0.092 & 0.196 & 0.609 & 0.359 & 0.584 & 0.060 \\
\hline Arcuate anterior segment $R$ & 0.780 & 0.308 & 0.007 & 0.958 & 0.437 & 0.890 \\
\hline Inferior fronto occipital fasciculus $L$ & 0.374 & 0.418 & 0.526 & 0.088 & 0.074 & 0.768 \\
\hline Inferior fronto occipital fasciculus $\mathbf{R}$ & 0.522 & 0.392 & 0.044 & 0.921 & 0.218 & 0.906 \\
\hline Corpus callosum & 0.066 & 0.000 & 0.000 & 0.556 & 0.749 & 0.052 \\
\hline Cortico-spinal L & 0.103 & 0.494 & 0.002 & 0.969 & 0.138 & 0.454 \\
\hline Cortico-spinal R & 0.031 & 0.721 & 0.009 & 0.457 & 0.551 & 0.419 \\
\hline Anterior thalamic radiations $L$ & 0.029 & 0.642 & 0.000 & 0.957 & 0.345 & 0.201 \\
\hline Anterior thalamic radiations $\mathbf{R}$ & 0.249 & 0.020 & 0.000 & 0.706 & 0.701 & 0.323 \\
\hline Fronto-striatal L & 0.131 & 0.970 & 0.021 & 0.995 & 0.339 & 0.102 \\
\hline Fronto-striatal R & 0.005 & 0.126 & 0.187 & 0.559 & 0.371 & 0.640 \\
\hline Fronto pontine $\mathrm{L}$ & 0.122 & 0.256 & 0.050 & 0.799 & 0.490 & 0.057 \\
\hline Fronto pontine $\mathbf{R}$ & 0.006 & 0.096 & 0.183 & 0.830 & 0.769 & 0.046 \\
\hline Paracentral $\mathrm{U}$ tract $\mathrm{L}$ & 0.456 & 0.680 & 0.398 & 0.917 & 0.910 & 0.109 \\
\hline Paracentral $\mathbf{U}$ tract $\mathbf{R}$ & 0.603 & 0.554 & 0.868 & 0.468 & 0.395 & 0.668 \\
\hline HandSup $U$ tract $L$ & 0.023 & 0.843 & 0.405 & 0.024 & 0.414 & 0.437 \\
\hline HandSup U tract R & 0.094 & 0.735 & 0.607 & 0.081 & 0.069 & 0.077 \\
\hline Handinf $U$ tract $L$ & 0.684 & 0.655 & 0.054 & 0.841 & 0.312 & 0.359 \\
\hline Handinf $U$ tract $R$ & 0.016 & 0.443 & 0.170 & 0.579 & 0.274 & 0.535 \\
\hline Handmid $U$ tract $L$ & 0.009 & 0.713 & 0.131 & 0.058 & 0.171 & 0.640 \\
\hline Handmid U tract $R$ & 0.030 & 0.793 & 0.797 & 0.909 & 0.429 & 0.163 \\
\hline Face $U$ tract $L$ & 0.739 & 0.645 & 0.367 & 0.976 & 0.736 & 0.508 \\
\hline Face $U$ tract $R$ & 0.698 & 0.215 & 0.920 & 0.840 & 0.566 & 0.325 \\
\hline Frontal aslant Tract $L$ & 0.410 & 0.428 & 0.006 & 0.330 & 0.457 & 0.339 \\
\hline Frontal aslant Tract $\mathbf{R}$ & 0.545 & 0.282 & 0.031 & 0.121 & 0.464 & 0.437 \\
\hline Fronto Insular tract $1 \mathrm{~L}$ & 0.978 & 0.450 & 0.305 & 0.420 & 0.440 & 0.479 \\
\hline Fronto Insular tract $1 \mathbf{R}$ & 0.567 & 0.790 & 0.498 & 0.239 & 0.145 & 0.570 \\
\hline Fronto Insular tract $2 \mathrm{~L}$ & 0.908 & 0.754 & 0.564 & 0.405 & 0.637 & 0.666 \\
\hline Fronto Insular tract 2 R & 0.841 & 0.734 & 0.384 & 0.587 & 0.047 & 0.030 \\
\hline Fronto Insular tract $3 \mathbf{L}$ & 0.353 & 0.431 & 0.618 & 0.336 & 0.447 & 0.123 \\
\hline Fronto Insular tract $3 \mathbf{R}$ & 0.565 & 0.191 & 0.247 & 0.196 & 0.829 & 0.225 \\
\hline Fronto Insular tract $4 \mathrm{~L}$ & 0.802 & 0.931 & 0.308 & 0.639 & 0.760 & 0.226 \\
\hline Fronto Insular tract $4 \mathrm{R}$ & 0.526 & 0.450 & 0.088 & 0.922 & 0.603 & 0.822 \\
\hline Fronto Insular tract $5 \mathrm{~L}$ & 0.531 & 0.015 & 0.010 & 0.238 & 0.760 & 0.356 \\
\hline Fronto Insular tract 5 R & 0.401 & 0.597 & 0.096 & 0.755 & 0.828 & 0.838 \\
\hline Frontal inferior longitudinal L & 0.065 & 0.256 & 0.000 & 0.061 & 0.728 & 0.058 \\
\hline Frontal inferior longitudinal $\mathbf{R}$ & 0.158 & 0.110 & 0.133 & 0.155 & 0.520 & 0.599 \\
\hline Frontal orbito polar $L$ & 0.229 & 0.629 & 0.000 & 0.526 & 0.607 & 0.042 \\
\hline Frontal orbito polar $\mathbf{R}$ & 0.022 & 0.417 & 0.191 & 0.411 & 0.032 & 0.988 \\
\hline Frontal superior longitudinal L & 0.181 & 0.644 & 0.088 & 0.672 & 0.501 & 0.117 \\
\hline Frontal superior longitudinal $\mathbf{R}$ & 0.284 & 0.258 & 0.891 & 0.613 & 0.646 & 0.312 \\
\hline Fronto Marginal tract $L$ & 0.102 & 0.647 & 0.859 & 0.501 & 0.043 & 0.250 \\
\hline Fronto Marginal tract $\mathbf{R}$ & 0.028 & 0.601 & 0.670 & 0.482 & 0.264 & 0.553 \\
\hline
\end{tabular}

